Geometry $8 \mathcal{T}$ Topology Monographs

Volume 7: Proceedings of the Casson Fest

Pages 431-491

\title{
Circular groups, planar groups, and the Euler class
}

\section{DANny CAlegari}

\begin{abstract}
We study groups of $C^{1}$ orientation-preserving homeomorphisms of the plane, and pursue analogies between such groups and circularlyorderable groups. We show that every such group with a bounded orbit is circularly-orderable, and show that certain generalized braid groups are circularly-orderable.

We also show that the Euler class of $C^{\infty}$ diffeomorphisms of the plane is an unbounded class, and that any closed surface group of genus $>1$ admits a $C^{\infty}$ action with arbitrary Euler class. On the other hand, we show that $\mathbb{Z} \oplus \mathbb{Z}$ actions satisfy a homological rigidity property: every orientationpreserving $C^{1}$ action of $\mathbb{Z} \oplus \mathbb{Z}$ on the plane has trivial Euler class. This gives the complete homological classification of surface group actions on $\mathbb{R}^{2}$ in every degree of smoothness.
\end{abstract}

AMS Classification 37C85; 37E30, 57M60

Keywords Euler class, group actions, surface dynamics, braid groups, $C^{1}$ actions

This paper is dedicated to Andrew Casson, on the occasion of his 60th birthday. Happy birthday, Andrew!

\section{Introduction}

We are motivated by the following question: what kinds of countable groups $G$ act on the plane? And for a given group $G$ known to act faithfully, what is the best possible analytic quality for a faithful action?

This is a very general problem, and it makes sense to narrow focus in order to draw useful conclusions. Groups can be sifted through many different kinds of strainers: finitely presented, hyperbolic, amenable, property $\mathrm{T}$, residually finite, etc. Here we propose that "acts on a circle" or "acts on a line" is an interesting sieve to apply to groups $G$ which act on the plane.

The theory of group actions on 1-dimensional manifolds is rich and profound, and has many subtle connections with algebra, logic, analysis, topology, ergodic 
theory, etc. One would hope that some of the depth of this theory would carry across to the study of group actions on 2-dimensional manifolds.

The most straightforward way to establish a connection between groups which act in 1 and 2 dimensions is to study when the groups acting in either case are abstractly isomorphic. Therefore we study subgroups $G<\mathrm{Homeo}^{+}\left(\mathbb{R}^{2}\right)$, and ask under what general conditions are they isomorphic (as abstract groups) to subgroups of $\mathrm{Homeo}^{+}\left(S^{1}\right)$.

One reason to compare the groups $\mathrm{Homeo}^{+}\left(S^{1}\right)$ and Homeo ${ }^{+}\left(\mathbb{R}^{2}\right)$ comes from their cohomology as discrete groups. A basic theorem of Mather and Thurston says that the cohomology of both of these groups, thought of as discrete groups, is equal, and is equal to $\mathbb{Z}[e]$ where $[e]$ in dimension 2 is free, and is the Euler class. Thus at a classical algebraic topological level, these groups are not easily distinguished, and we should not be surprised if many subgroups of $\mathrm{Homeo}^{+}\left(\mathbb{R}^{2}\right)$ can be naturally made to act faithfully on a circle. We establish that countable $C^{1}$ groups of homeomorphisms of the plane which satisfy a certain dynamical condition - that they have a bounded orbit - are all isomorphic to subgroups of $\mathrm{Homeo}^{+}\left(S^{1}\right)$.

On the other hand, the bounded cohomology of these groups is dramatically different. The classical Milnor-Wood inequality says that the Euler class on Homeo $^{+}\left(S^{1}\right)$ is a bounded class. By contrast, the Euler class on $\mathrm{Homeo}^{+}\left(\mathbb{R}^{2}\right)$ is unbounded. This was known to be true for $C^{0}$ homeomorphisms; in this paper we establish that it is also true for $C^{\infty}$ homeomorphisms. However, a surprising rigidity phenomenon manifests itself: for $C^{1}$ actions of $\mathbb{Z} \oplus \mathbb{Z}$ we show that the Euler class must always vanish, which would be implied by boundedness. This is surprising for two reasons: firstly, because we show that the Euler class can take on any value for $C^{\infty}$ actions of higher genus surface groups, and secondly because the Euler class can take on any value for $C^{0}$ actions of $\mathbb{Z} \oplus \mathbb{Z}$. It would be very interesting to understand the full range of this homological rigidity.

We now turn to a more precise statement of results.

\subsection{Statement of results}

Section 2 contains background material on left-orderable and circularly-orderable groups. This material is all standard, and is collected there for the convenience of the reader. The main results there are that a countable group is left-orderable iff it admits an injective homomorphism to $\mathrm{Homeo}^{+}(\mathbb{R})$, and circularly-orderable iff it admits an injective homomorphism to $\operatorname{Homeo}^{+}\left(S^{1}\right)$. 
Thus, group actions on 1-manifolds can be characterized in purely algebraic terms. The expert may feel free to skip Section 2 and move on to Section 3.

Section 3 concerns $C^{1}$ subgroups $G<\operatorname{Homeo}^{+}\left(\mathbb{R}^{2}\right)$ with bounded orbits. Our first main result is a generalization of a theorem of Dehornoy [7] about orderability of the usual braid groups.

For $C$ a compact, totally disconnected subset of the open unit disk $D$, we use the notation $B_{C}$ to denote the group of homotopy classes of homeomorphisms of $\bar{D} \backslash C$ to itself which are fixed on the boundary, and $B_{C}^{\prime}$ to denote the group of homotopy classes of (orientation-preserving) homeomorphisms which might or might not be fixed on the boundary. Informally, $B_{C}$ is the "braid group" of $C$. In particular, if $C$ consists of $n$ isolated points, $B_{C}$ is the usual braid group on $n$ strands.

Theorem A Let $C$ be a compact, totally disconnected subset of the open unit disk $D$. Then $B_{C}^{\prime}$ is circularly-orderable, and $B_{C}$ is left-orderable.

Using this theorem and the Thurston stability theorem [37], we show the following:

Theorem B Let $G$ be a group of orientation preserving $C^{1}$ homeomorphisms of $\mathbb{R}^{2}$ with a bounded orbit. Then $G$ is circularly-orderable.

Section 4 concerns the Euler class for planar actions. As intimated above, we show that the Euler class can take on any value for $C^{\infty}$ actions of higher genus surface groups:

Theorem $\mathbf{C}$ For each integer $i$, there is a $C^{\infty}$ action

$$
\rho_{i}: \pi_{1}(S) \rightarrow \text { Diffeo }^{+}\left(\mathbb{R}^{2}\right)
$$

where $S$ denotes the closed surface of genus 2, satisfying

$$
\rho_{i}^{*}([e])([S])=i .
$$

In particular, the Euler class $[e] \in H^{2}\left(\right.$ Diffeo $\left.^{+}\left(\mathbb{R}^{2}\right) ; \mathbb{Z}\right)$ is unbounded.

This answers a question of Bestvina.

Using this result, we are able to construct examples of finitely-generated torsionfree groups of orientation-preserving homeomorphisms of $\mathbb{R}^{2}$ which are not circularly-orderable, thereby answering a question of Farb.

It might seem from this theorem that there are no homological constraints on group actions on $\mathbb{R}^{2}$, but in fact for $C^{1}$ actions, we show the following: 
Theorem D Let $\rho: \mathbb{Z} \oplus \mathbb{Z} \rightarrow$ Homeo $^{+}\left(\mathbb{R}^{2}\right)$ be a $C^{1}$ action. Then the Euler class $\rho^{*}([e]) \in H^{2}(\mathbb{Z} \oplus \mathbb{Z} ; \mathbb{Z})$ is zero.

It should be emphasized that this is not a purely local theorem, but uses in an essential way Brouwer's famous theorem on fixed-point-free orientation-preserving homeomorphisms of $\mathbb{R}^{2}$.

Together with an example of Bestvina, theorems $\mathrm{C}$ and $\mathrm{D}$ give a complete homological classification of (orientation-preserving) actions of (oriented) surface groups on $\mathbb{R}^{2}$ in every degree of smoothness.

\section{$1.2 \quad$ Acknowledgements}

I would like to thank Mladen Bestvina, Nathan Dunfield, Bob Edwards, Benson Farb, John Franks, Étienne Ghys, Michael Handel, Dale Rolfsen, Frédéric le Roux, Takashi Tsuboi, Amie Wilkinson and the anonymous referee for some very useful conversations and comments.

I would especially like to single out Étienne Ghys for thanks, for reading an earlier version of this paper and providing me with copious comments, observations, references, and counterexamples to some naive conjectures.

While writing this paper, I received partial support from the Sloan foundation, and from NSF grant DMS-0405491.

\section{$2 \quad$ Left-orderable groups and circular groups}

In this section we define left-orderable and circularly-orderable groups, and present some of their elementary properties. None of the material in this section is new, but perhaps the exposition will be useful to the reader. Details and references can be found in [29], [38], [11], [21] and [20], as well as other papers mentioned in the text as appropriate.

\subsection{Left-invariant orders}

Definition 2.1.1 Let $G$ be a group. A left-invariant order on $G$ is a total order $<$ such that, for all $\alpha, \beta, \gamma$ in $G$,

$$
\alpha<\beta \text { iff } \gamma \alpha<\gamma \beta \text {. }
$$

A group which admits a left-invariant order is said to be left-orderable. 
We may sometimes abbreviate "left-orderable" to LO. Note that a left-orderable group may admit many distinct left-invariant orders. For instance, the group $\mathbb{Z}$ admits exactly two left-invariant orders.

The following lemma gives a characterization of left-orderable groups:

Lemma 2.1.2 A group $G$ admits a left-invariant order iff there is a disjoint partition of $G=P \cup N \cup I d$ such that $P \cdot P \subset P$ and $P^{-1}=N$.

Proof If $G$ admits a left-invariant order, set $P=\{g \in G: g>$ Id $\}$. Conversely, given a partition of $G$ into $P, N$, Id with the properties above, we can define a left-invariant order by setting $h<g$ iff $h^{-1} g \in P$.

Notice that Lemma 2.1.2 implies that any nontrivial LO group is infinite, and torsion-free. Notice also that any partition of $G$ as in Lemma 2.1.2 satisfies $N \cdot N \subset N$. For such a partition, we sometimes refer to $P$ and $N$ as the positive and negative cone of $G$ respectively.

LO is a local property. That is to say, it depends only on the finitely-generated subgroups of $G$. We make this precise in the next two lemmas. First we show that if a group fails to be left-orderable, this fact can be verified by examining a finite subset of the multiplication table for the group, and applying the criterion of Lemma 2.1.2.

Lemma 2.1.3 A group $G$ is not left-orderable iff there is some finite symmetric subset $S=S^{-1}$ of $G$ with the property that for every disjoint partition $S \backslash I d=$ $P_{S} \cup N_{S}$, one of the following two properties holds:

(1) $P_{S} \cap P_{S}^{-1} \neq \emptyset$ or $N_{S} \cap N_{S}^{-1} \neq \emptyset$

(2) $\left(P_{S} \cdot P_{S}\right) \cap N_{S} \neq \emptyset$ or $\left(N_{S} \cdot N_{S}\right) \cap P_{S} \neq \emptyset$

Proof It is clear that the existence of such a subset contradicts Lemma 2.1.2. So it suffices to show the converse.

The set of partitions of $G \backslash \mathrm{Id}$ into disjoint sets $P, N$ is just $2^{G \backslash \mathrm{Id}}$, which is compact with the product topology by Tychonoff's theorem. By abuse of notation, if $\pi \in 2^{G \backslash \mathrm{Id}}$ and $g \in G \backslash \mathrm{Id}$, we write $\pi(g)=P$ or $\pi(g)=N$ depending on whether the element $g$ is put into the set $P$ or $N$ under the partition corresponding to $\pi$.

For every element $\alpha \in G \backslash \mathrm{Id}$, define $A_{\alpha}$ to be the open subset of $2^{G \backslash \mathrm{Id}}$ for which $\pi(\alpha)=\pi\left(\alpha^{-1}\right)$. For every pair of elements $\alpha, \beta \in G \backslash \operatorname{Id}$ with $\alpha \neq \beta^{-1}$, define $B_{\alpha, \beta}$ to be the open subset of $2^{G \backslash \text { Id }}$ for which $\pi(\alpha)=\pi(\beta)$ but $\pi(\alpha) \neq \pi(\alpha \beta)$. 
Now, if $G$ is not LO, then by Lemma 2.1.2, every partition $\pi \in 2^{G \backslash \mathrm{Id}}$ is contained in some $A_{\alpha}$ or $B_{\alpha, \beta}$. That is, the sets $A_{\alpha}, B_{\alpha, \beta}$ are an open cover of $2^{G \backslash \mathrm{Id}}$. By compactness, there is some finite subcover. Let $S$ denote the set of indices of the sets $A_{\alpha}, B_{\alpha, \beta}$ appearing in this finite subcover, together with their inverses. Then $S$ satisfies the statement of the lemma.

Remark 2.1.4 An equivalent statement of this lemma is that for a group $G$ which is not LO, there is a finite subset $S=\left\{g_{1}, \cdots, g_{n}\right\} \subset G \backslash$ Id with $S \cap S^{-1}=\emptyset$ such that for all choices of signs $e_{i} \in \pm 1$, the semigroup generated by the $g_{i}^{e_{i}}$ contains Id.

To see this, observe that a choice of sign $e_{i} \in \pm 1$ amounts to a choice of partition of $S \cup S^{-1}$ into $P_{S}$ and $N_{S}$. Then if $G$ is not LO, the semigroup of positive products of the $P_{S}$ must intersect the semigroup of positive products of the $N_{S}$; that is, $p=n$ for $p$ in the semigroup generated by $P_{S}$ and $n$ in the semigroup generated by $N_{S}$. But this implies $n^{-1}$ is in the semigroup generated by $P_{S}$, and therefore so too is the product $n^{-1} p=\mathrm{Id}$.

Remark 2.1.5 Given a finite symmetric subset $S$ of $G$ and a multiplication table for $G$, one can check by hand whether the set $S$ satisfies the hypotheses of Lemma 2.1.3. It follows that if $G$ is a group for which there is an algorithm to solve the word problem, then if $G$ is not left-orderable, one can certify that $G$ is not left-orderable by a finite combinatorial certificate.

The next lemma follows easily from Lemma 2.1.3:

Lemma 2.1.6 A group $G$ is left-orderable iff every finitely-generated subgroup is left-orderable.

Proof We use the $A, B$ notation from Lemma 2.1.3.

Observe that a left-ordering on $G$ restricts to a left-ordering on any finitelygenerated subgroup $H<G$.

Conversely, suppose $G$ is not left-orderable. By Lemma 2.1.3 we can find a finite set $S$ satisfying the hypotheses of that lemma. Let $H$ be the group generated by $S$. Then Lemma 2.1.3 implies that $H$ is not left-orderable.

Remark 2.1.7 To see this in more topological terms: observe that there is a restriction map

$$
\text { res: } 2^{G \backslash \mathrm{Id}} \rightarrow 2^{H \backslash \mathrm{Id}}
$$


which is surjective, and continuous with respect to the product topologies. It follows that the union of the sets $\operatorname{res}\left(A_{\alpha}\right), \operatorname{res}\left(B_{\alpha, \beta}\right)$ with $\alpha, \beta \in S$ is an open cover of $2^{H \backslash \mathrm{Id}}$, and therefore $H$ is not left-orderable.

We now study homomorphisms between LO groups.

Definition 2.1.8 Let $S$ and $T$ be totally-ordered sets. A map $\phi: S \rightarrow T$ is monotone if for every pair $s_{1}, s_{2} \in S$ with $s_{1}>s_{2}$, either $\phi\left(s_{1}\right)>\phi\left(s_{2}\right)$ or $\phi\left(s_{1}\right)=\phi\left(s_{2}\right)$.

Let $G$ and $H$ be left-orderable groups, and choose a left-invariant order on each of them. A homomorphism $\phi: G \rightarrow H$ is monotone if it is monotone as a map or totally-ordered sets.

LO behaves well under short exact sequences:

Lemma 2.1.9 Suppose $K, H$ are left-orderable groups, and suppose we have a short exact sequence

$$
0 \longrightarrow K \longrightarrow G \longrightarrow H \longrightarrow 0 .
$$

Then for every left-invariant order on $K$ and $H$, the group $G$ admits a leftinvariant order compatible with that of $K$, such that the surjective homomorphism to $H$ is monotone.

Proof Let $\phi: G \rightarrow H$ be the homomorphism implicit in the short exact sequence. The order on $G$ is uniquely determined by the properties that it is required to satisfy:

(1) If $\phi\left(g_{1}\right) \neq \phi\left(g_{2}\right)$ then $g_{1}>g_{2}$ in $G$ iff $\phi\left(g_{1}\right)>\phi\left(g_{2}\right)$ in $H$

(2) If $\phi\left(g_{1}\right)=\phi\left(g_{2}\right)$ then $g_{2}^{-1} g_{1} \in K$, so $g_{1}>g_{2}$ in $G$ iff $g_{2}^{-1} g_{1}>$ Id in $K$

This defines a total order on $G$ and is left-invariant, as required.

Definition 2.1.10 A group $G$ is locally $L O$-surjective if every finitely-generated subgroup $H$ admits a surjective homomorphism $\phi_{H}: H \rightarrow L_{H}$ to an infinite LO group $L_{H}$.

A group $G$ is locally indicable if every finitely-generated subgroup $H$ admits a surjective homomorphism to $\mathbb{Z}$. In particular, a locally indicable group is locally LO-surjective, though the converse is not true.

The following theorem is proved in [4]. We give a sketch of a proof. 
Theorem 2.1.11 (Burns-Hale) Suppose $G$ is locally LO-surjective. Then $G$ is $L O$.

Proof Suppose $G$ is locally LO-surjective but not LO. Then by Remark 2.1.4, there is some finite subset $\left\{g_{1}, \ldots, g_{n}\right\} \subset G \backslash \mathrm{Id}$ such that, for all choices of signs $e_{i} \in \pm 1$, the semigroup of positive products of the elements $g_{i}^{e_{i}}$ contains Id. Choose a set of such $g_{i}$ such that $n$ is smallest possible (obviously, $n \geq 2$ ). Let $G^{\prime}=\left\langle g_{1}, \ldots, g_{n}\right\rangle$. Then $G^{\prime}$ is finitely-generated. Since $G$ is locally LOsurjective, $G^{\prime}$ admits a surjective homomorphism to an infinite LO group

$$
\varphi: G^{\prime} \rightarrow H
$$

with kernel $K$. By the defining property of the $\left\{g_{i}\right\}$, at least one $g_{i}$ is in $K$ since otherwise there exist choices of signs $e_{i} \in \pm 1$ such that $\varphi\left(g_{i}^{e_{i}}\right)$ is in the positive cone of $H$, and therefore the same is true for the semigroup of positive products of such elements. But this would imply that the semigroup of positive products of the $g_{i}^{e_{i}}$ does not contain Id in $G^{\prime}$, contrary to assumption. Furthermore, since $H$ is nontrivial and $\varphi$ is surjective, at least one $g_{j}$ is not in $K$.

Reorder the indices of the $g_{i}$ so that $g_{1}, \ldots, g_{k} \notin K$ and $g_{k+1}, \ldots, g_{n} \in K$. Let $P(H)$ denote the positive elements of $H$. Since the $g_{i}$ with $i \leq k$ are not in $K$, it follows that there are choices $\delta_{1}, \ldots, \delta_{k} \in \pm 1$ such that $\varphi\left(g_{i}^{\delta_{i}}\right) \in P(H)$. Moreover, since $n$ was chosen to be minimal, there exist choices $\delta_{k+1}, \ldots, \delta_{n} \in$ \pm 1 such that no positive product of elements of $g_{k+1}^{\delta_{k+1}}, \ldots, g_{n}^{\delta_{n}}$ is equal to Id.

On the other hand, by the definition of $g_{i}$, there are positive integers $n_{i}$ such that

$$
\mathrm{Id}=g_{i(1)}^{n_{1} \delta_{i(1)}} \cdots g_{i(s)}^{n_{s} \delta_{i(s)}}
$$

where each $i(j)$ is between 1 and $n$. By hypothesis, $i(j) \leq k$ for at least one $j$. But this implies that the image of the right hand side of this equation under $\varphi$ is in $P(H)$, which is a contradiction.

Theorem 2.1.11 has the corollary that a locally indicable group is LO. It is this corollary that will be most useful to us.

\subsection{Circular orders}

The approach we take in this section is modelled on [38], although an essentially equivalent approach is found in [11]. 
We first define a circular ordering on a set. Suppose $p$ is a point in an oriented circle $S^{1}$. Then $S^{1} \backslash p$ is homeomorphic to $\mathbb{R}$, and the orientation on $\mathbb{R}$ defines a natural total order on $S^{1} \backslash p$. In general, a circular order on a set $S$ is defined by a choice of total ordering on each subset of the form $S \backslash p$, subject to certain compatibility conditions which we formalize below.

Definition 2.2.1 Let $S$ be a set. A circular ordering on a set $S$ with at least 4 elements is a choice of total ordering on $S \backslash p$ for every $p \in S$, such that if $<_{p}$ is the total ordering defined by $p$, and $p, q \in S$ are two distinct elements, the total orderings $<_{p},<_{q}$ differ by a cut on their common domain of definition. That is, for any $x, y$ distinct from $p, q$, the order of $x$ and $y$ with respect to $<_{p}$ and $<_{q}$ is the same unless $x<_{p} q<_{p} y$, in which case we have $y<_{q} p<_{q} x$. We also say that the order $<_{q}$ on $S \backslash\{p, q\}$ is obtained from the order $<_{p}$ on $S \backslash p$ by cutting at $q$.

If $S$ has exactly 3 elements $S=\{x, y, z\}$, we must add the condition that $y<_{x} z$ iff $z<_{y} x$. Note that this condition is implied by the condition in the previous paragraph if $S$ has at least 4 elements.

To understand the motivation for the terminology, consider the operation of cutting a deck of cards.

Example 2.2.2 The oriented circle $S^{1}$ is circularly-ordered, where for any $p$, the ordering $<_{p}$ is just the ordering on $S^{1} \backslash p \cong \mathbb{R}$ induced by the orientation on $\mathbb{R}$.

Definition 2.2.3 A set with three elements $x, y, z$ admits exactly two circular orders, depending on whether $y<_{x} z$ or $z<_{x} y$. In the first case, we say the triple $(x, y, z)$ is positively-ordered and in the second case, we say it is negativelyordered.

We also refer to a positively-ordered triple of points as anticlockwise and a negatively-ordered triple as clockwise, by analogy with the standard circular order on triples of points in the positively oriented circle.

A circular ordering on a set $S$ induces a circular ordering on any subset $T \subset S$. If $T_{\alpha}$ is a family of subsets of $S$ which are all circularly-ordered, we say the circular orderings on the $T_{\alpha}$ are compatible if they are simultaneously induced by some circular ordering on $S$.

It is clear that a circular ordering on a set $S$ is determined by the family of circular orderings on all triples of elements in $S$. Conversely, the following 
lemma characterizes those families of circular orderings on triples of elements which arise from a circular ordering on all of $S$ :

Lemma 2.2.4 Suppose $S$ is a set. A circular ordering on all triples of distinct elements on $S$ is compatible iff for every subset $Q \subset S$ with four elements, the circular ordering on triples of distinct elements of $Q$ is compatible. In this case, these circular orderings are uniquely compatible, and determine a circular ordering on $S$.

Proof A circular ordering on triples in $S$ defines, for any $p \in S$, a binary relation $<_{p}$ on $S \backslash p$ by $x<_{p} y$ iff the triple $(p, x, y)$ is positively-ordered. To see that this binary relation defines a total ordering on $S \backslash p$, we must check transitivity of $<_{p}$. But this follows from compatibility of the circular ordering on quadruples $Q$. It is straightforward to check that the total orders $<_{p}$ and $<_{q}$ defined in this way differ by a cut for distinct $p, q$.

Definition 2.2.5 Let $C_{1}, C_{2}$ be circularly-ordered sets. A map $\phi: C_{1} \rightarrow C_{2}$ is monotone if for each $c \in C_{2}$ and each $d \in \phi^{-1}(c)$, the restriction map between totally-ordered sets

$$
\phi:\left(C_{1} \backslash \phi^{-1}(c),<_{d}\right) \rightarrow\left(C_{2} \backslash c,<_{c}\right)
$$

is monotone.

There is a natural topology on a circularly-ordered set for which monotone maps are continuous.

Definition 2.2.6 Let $O,<$ be a totally-ordered set. The order topology on $O$ is the topology generated by open sets of the form $\{x \mid x>p\}$ and $\{x \mid x<p\}$ for all $p \in O$. Let $S$ be a circularly-ordered set. The order topology on $S$ is the topology generated on each $S \backslash p$ by the (usual) order topology on the totally-ordered set $S \backslash p,<_{p}$.

We now turn to the analogue of left-ordered groups for circular orderings.

Definition 2.2.7 A group $G$ is left circularly-ordered if it admits a circular order as a set which is preserved by the action of $G$ on itself on the left. A group is left circularly-orderable if it can be left circularly-ordered.

We usually abbreviate this by saying that a group is circularly-orderable if it admits a circular order. 
Example 2.2.8 A left-orderable group $G,<$ is circularly-orderable as follows: for each element $g \in G$, the total order $<_{g}$ on $G \backslash g$ is obtained from the total order $<$ by cutting at $g$.

Definition 2.2.9 The group of orientation-preserving homeomorphisms of $\mathbb{R}$ is denoted $\mathrm{Homeo}^{+}(\mathbb{R})$. The group of orientation-preserving homeomorphisms of the circle is denoted Homeo ${ }^{+}\left(S^{1}\right)$.

An action of $G$ on $\mathbb{R}$ or the circle by orientation-preserving homeomorphisms is the same thing as a representation in $\operatorname{Homeo}^{+}(\mathbb{R})$ or $\mathrm{Homeo}^{+}\left(S^{1}\right)$. We will see that for countable groups $G$, being LO is the same as admitting a faithful representation in $\mathrm{Homeo}^{+}(\mathbb{R})$, and $\mathrm{CO}$ is the same as admitting a faithful representation in $\mathrm{Homeo}^{+}\left(S^{1}\right)$. First we give one direction of the implication.

Lemma 2.2.10 If $G$ is countable and admits a left-invariant circular order, then $G$ admits a faithful representation in $\mathrm{Homeo}^{+}\left(S^{1}\right)$.

Proof Let $g_{i}$ be a countable enumeration of the elements of $G$. We define an embedding $e: G \rightarrow S^{1}$ as follows. The first two elements $g_{1}, g_{2}$ map to arbitrary distinct points in $S^{1}$. Thereafter, we use the following inductive procedure to uniquely extend $e$ to each $g_{n}$.

Firstly, for every $n>2$, the map

$$
e: \bigcup_{i \leq n} g_{i} \longrightarrow \bigcup_{i \leq n} e\left(g_{i}\right)
$$

should be injective and circular-order-preserving, where the $e\left(g_{i}\right)$ are circularly-ordered by the natural circular ordering on $S^{1}$. Secondly, for every $n>$ 2 , the element $e\left(g_{n}\right)$ should be taken to the midpoint of the unique interval complementary to $\bigcup_{i<n} e\left(g_{i}\right)$ compatible with the first condition. This defines $e\left(g_{n}\right)$ uniquely, once $e\left(g_{i}\right)$ has been defined for all $i<n$.

It is easy to see that the left action of $G$ on itself extends uniquely to a continuous order preserving homeomorphism of the closure $\overline{e(G)}$ to itself. The complementary intervals $I_{i}$ to $\overline{e(G)}$ are permuted by the action of $G$; we choose an identification $\varphi_{i}: I_{i} \rightarrow I$ of each interval with $I$, and extend the action of $G$ so that if $g\left(I_{i}\right)=I_{j}$ then the action of $g$ on $I_{i}$ is equal to

$$
\left.g\right|_{I_{i}}=\varphi_{j}^{-1} \varphi_{i} .
$$

This defines a faithful representation of $G$ in $\mathrm{Homeo}^{+}\left(S^{1}\right)$, as claimed. 
Remark 2.2.11 Note that basically the same argument shows that a leftorderable countable group is isomorphic to a subgroup of $\mathrm{Homeo}^{+}(\mathbb{R})$. Notice further that this construction has an important property: if $G$ is a countable left- or circularly-ordered group, then $G$ is circular or acts on $\mathbb{R}$ in such a way that some point has trivial stabilizer. In particular, any point in the image of $e$ has trivial stabilizer.

Short exact sequences intertwine circularity and left-orderability:

Lemma 2.2.12 Suppose

$$
0 \longrightarrow K \longrightarrow G \longrightarrow H \longrightarrow 0
$$

is a short exact sequence, where $K$ is left-ordered and $H$ is circularly-ordered. Then $G$ can be circularly-ordered in such a way that the inclusion of $K$ into $G$ respects the order on $G \backslash g$ for any $g$ not in $K$, and the map from $G$ to $H$ is monotone.

Proof Let $\phi: G \rightarrow H$ be the homomorphism in the short exact sequence. Let $g_{1}, g_{2}, g_{3}$ be three distinct elements of $G$. We define the circular order as follows:

(1) If $\phi\left(g_{1}\right), \phi\left(g_{2}\right), \phi\left(g_{3}\right)$ are distinct, circularly-order them by the circular order on their image in $H$

(2) If $\phi\left(g_{1}\right)=\phi\left(g_{2}\right)$ but these are distinct from $\phi\left(g_{3}\right)$, then $g_{2}^{-1} g_{1} \in K$. If $g_{2}^{-1} g_{1}<$ Id then $g_{1}, g_{2}, g_{3}$ is positively-ordered, otherwise it is negativelyordered

(3) If $\phi\left(g_{1}\right)=\phi\left(g_{2}\right)=\phi\left(g_{3}\right)$ then $g_{3}^{-1} g_{1}, g_{3}^{-1} g_{2}$, Id are all in $K$, and therefore inherit a total ordering. The three corresponding elements of $G$ in the same total order are negatively-ordered

One can check that this defines a left-invariant circular order on $G$.

Here our convention has been that the orientation-preserving inclusion of $\mathbb{R}$ into $S^{1} \backslash p$ is order-preserving.

We will show that for countable groups, being $\mathrm{LO}$ or $\mathrm{CO}$ is equivalent to admitting a faithful representation in $\mathrm{Homeo}^{+}(\mathbb{R})$ or $\mathrm{Homeo}^{+}\left(S^{1}\right)$ respectively. But first we must describe an operation due to Denjoy [9] of blowing up or Denjoying an action. 
Construction 2.2.13 (Denjoy) Let $\rho: G \rightarrow \operatorname{Homeo}^{+}\left(S^{1}\right)$ be an action of a countable group on $S^{1}$. For convenience, normalize $S^{1}$ to have length 1 . Let $p \in S^{1}$ be some point. Let $O$ denote the countable orbit of $p$ under $G$, and let $\phi: O \rightarrow \mathbb{R}^{+}$assign a positive real number to each $o \in O$ such that $\sum_{o \in O} \phi(o)=1$. Choose some point $q$ not in $O$, and define $\tau:[0,1] \rightarrow S^{1}$ to be an orientation-preserving parametrization by length, which takes the two endpoints to $q$. Define $\sigma:[0,1] \rightarrow[0,2]$ by

$$
\sigma(t)=t+\sum_{o \in O: \tau^{-1}(o) \leq t} \phi(o) .
$$

Then $\sigma$ is discontinuous on $\tau^{-1}(O)$, and its graph can be completed to a continuous image of $I$ in $[0,1] \times[0,2]$ by adding a vertical segment of length $\phi(o)$ at each point $\tau^{-1}(o)$ where $o \in O$. Identify opposite sides of $[0,1] \times[0,2]$ to get a torus, in which the closure of the graph of $\sigma$ closes up to become a $(1,1)$ curve which, by abuse of notation, we also refer to as $\sigma$. Notice that projection $\pi_{h}$ onto the horizontal factor defines a monotone map from $\sigma$ to $S^{1}$.

Then the action of $G$ on $S^{1}$ extends in an obvious way to an action on this torus which leaves the $(1,1)$ curve invariant, and also preserves the foliations of the torus by horizontal and vertical curves. Up to conjugacy in $\mathrm{Homeo}^{+}(\sigma)$, the action of $G$ on $\sigma$ is well-defined, and is called the blown-up action at $p$. The pushforward of this blown-up action under $\left(\pi_{h}\right)_{*}$ recovers the original action of $G$ on $S^{1}$; that is, the two actions are related by a degree one monotone map, and are said to be semi-conjugate. The equivalence relation that this generates is called monotone equivalence.

With this construction available to us, we demonstrate the equivalence of $\mathrm{CO}$ with admitting a faithful representation in $\mathrm{Homeo}^{+}\left(S^{1}\right)$.

Theorem 2.2.14 A countable group $G$ is left- or circularly-ordered iff $G$ admits a faithful homomorphism to $\mathrm{Homeo}^{+}(\mathbb{R})$ or $\mathrm{Homeo}^{+}\left(S^{1}\right)$ respectively. Moreover, the action on $\mathbb{R}$ or $S^{1}$ can be chosen so that some point has a trivial stabilizer.

Proof In Lemma 2.2.10 we have already showed how a left or circular order gives rise to a faithful action on $\mathbb{R}$ or $S^{1}$. So it remains to prove the converse.

Let $\phi: G \rightarrow \operatorname{Homeo}^{+}(\mathbb{R})$ be faithful. Let $p_{i}$ be some sequence of points such that the intersection of the stabilizers of the $p_{i}$ is the identity. Some such sequence $p_{i}$ exists, since $G$ is countable, and any nontrivial element acts nontrivially at some point. Then each $p_{i}$ determines a (degenerate) left-invariant 
order on $G$, by setting $g>_{i} h$ if $g\left(p_{i}\right)>h\left(p_{i}\right)$, and $g={ }_{i} h$ if $g\left(p_{i}\right)=h\left(p_{i}\right)$. Then we define $g>h$ if $g>{ }_{i} h$ for some $i$, and $g={ }_{j} h$ for all $j<i$.

The definition of a circular order is similar: pick some point $p \in S^{1}$, and suppose that the stabilizer $\operatorname{stab}(p)$ is nontrivial. Then $\operatorname{stab}(p)$ acts faithfully on $S^{1}-p=\mathbb{R}$, so by the argument above, $\operatorname{stab}(p)$ is left-orderable and acts on $\mathbb{R}$. In fact, we know $\operatorname{stab}(p)$ acts on $\mathbb{R}$ in such a way that some point has trivial stabilizer. Let $\varphi: \operatorname{stab}(p) \rightarrow \operatorname{Homeo}^{+}(\mathbb{R})$ be such a representation. We construct a new representation $\phi^{\prime}: G \rightarrow \operatorname{Homeo}^{+}\left(S^{1}\right)$ from $\phi$ by blowing up $p$ as in Construction 2.2.13. The representation $\phi^{\prime}$ is monotone equivalent to $\phi$; that is, there is a monotone map $\pi: S^{1} \rightarrow S^{1}$ satisfying

$$
\pi_{*} \phi^{\prime}=\phi .
$$

Let $C \subset S^{1}$ be the set where the monotone map $\pi$ is not locally constant. We will modify the action of $G$ on $S^{1} \backslash C$ as follows. Note that $G$ acts on $C$ by the pullback under $\pi$ of the action on $S^{1}$ by $\phi$. We extend this action to $S^{1} \backslash C$ to define $\phi^{\prime \prime}$. Let $I$ be the open interval obtained by blowing up $p$. We identify $I$ with $\mathbb{R}$, and then let $\operatorname{stab}(p)$ act on $I$ by the pullback of $\varphi$ under this identification. Each other component $I_{i}$ in $S^{1} \backslash C$ is of the form $g(I)$ for some $g \in G$. Choose such a $g_{i}$ for each $I_{i}$, and pick an arbitrary (orientation preserving) identification $\varphi_{i}: I \rightarrow I_{i}$, and define $\left.\phi^{\prime \prime}\left(g_{i}\right)\right|_{I}=\varphi_{i}$. Now, for any $g \in G$, define $\left.g\right|_{I_{i}}$ as follows: suppose $g\left(I_{i}\right)=I_{j}$. Then $g_{j}^{-1} g g_{i} \in \operatorname{stab}(p)$, so define

$$
\left.\phi^{\prime \prime}(g)\right|_{I_{i}}=\varphi_{j} \varphi\left(g_{j}^{-1} g g_{i}\right) \varphi_{i}^{-1}: I_{i} \rightarrow I_{j} .
$$

It is clear that this defines a faithful representation $\phi^{\prime \prime}: G \rightarrow \operatorname{Homeo}^{+}\left(S^{1}\right)$, monotone equivalent to $\phi$, with the property that some point $q \in S^{1}$ has trivial stabilizer.

Now define a circular order on distinct triples $g_{1}, g_{2}, g_{3}$ by restricting the circular order on $S^{1}$ to the triple $g_{1}(q), g_{2}(q), g_{3}(q)$.

Notice that in this theorem, in order to recover a left or circular order on $G$ from a faithful action, all we used about $\mathbb{R}$ and $S^{1}$ was that they were ordered and circularly-ordered sets respectively.

With this theorem, and our lemmas on short exact sequences, we can deduce the existence of left or circular orders on countable groups from the existence of actions on ordered or circularly-ordered sets, with left-orderable kernel.

Theorem 2.2.15 Suppose a countable group $G$ admits an action by order preserving maps on a totally-ordered or circularly-ordered set $S$ in such a way 
that the kernel $K$ is left-orderable. Then $G$ admits a faithful, order preserving action on $\mathbb{R}$ or $S^{1}$, respectively.

Proof We discuss the case that $S$ is circularly-ordered, since this is slightly more complicated. Since $G$ is countable, it suffices to look at an orbit of the action, which will also be countable. By abuse of this notation, we also denote the orbit by $S$. As in Lemma 2.2.10, the set $S$ with its order topology is naturally order-isomorphic to a subset of $S^{1}$. Let $\bar{S}$ denote the closure of $S$ under this identification. Then the action of $G$ on $S$ extends to an orientation-preserving action on $S^{1}$, by permuting the complementary intervals to $\bar{S}$. It follows that the image of $G$ in $\mathrm{Homeo}^{+}\left(S^{1}\right)$ is CO, with kernel $K$. By Lemma 2.2.12, $G$ is CO. By Theorem 2.2.14, the proof follows.

The construction for $S$ totally-ordered is similar.

\subsection{Homological characterization of circular groups}

Circular orders on groups $G$ can be characterized homologically. There are at least two different ways of doing this, due to Thurston and Ghys respectively, which reflect two different ways of presenting the theory of group cohomology.

First, we recall the definition of group cohomology. For details, we refer to [21] or $[20]$.

Definition 2.3.1 Let $G$ be a group. The homogeneous chain complex of $G$ is a complex $C_{*}(G)_{h}$ where $C_{n}(G)_{h}$ is the free abelian group generated by equivalence classes of $(n+1)$-tuples $\left(g_{0}: g_{1}: \cdots: g_{n}\right)$, where two such tuples are equivalent if they are in the same coset of the left diagonal action of $G$ on the coordinates. That is,

$$
\left(g_{0}: g_{1}: \cdots: g_{n}\right) \sim\left(g g_{0}: g g_{1}: \cdots: g g_{n}\right) .
$$

The boundary operator in homogeneous coordinates is very simple, defined by the formula

$$
\partial\left(g_{0}: \cdots: g_{n}\right)=\sum_{i=0}^{n}(-1)^{i}\left(g_{0}: \cdots: \widehat{g}_{i}: \cdots: g_{n}\right) \text {. }
$$

The inhomogeneous chain complex of $G$ is a complex $C_{*}(G)_{i}$ where $C_{n}(G)_{i}$ is the free abelian group generated by $n$-tuples $\left(f_{1}, \ldots, f_{n}\right)$. The boundary 
operator in inhomogeneous coordinates is more complicated, defined by the formula

$$
\begin{aligned}
\partial\left(f_{1}, \ldots, f_{n}\right) & =\left(f_{2}, \ldots, f_{n}\right)+\sum_{i=1}^{n-1}(-1)^{i}\left(f_{1}, \ldots, f_{i} f_{i+1}, \ldots, f_{n}\right) \\
& +(-1)^{n}\left(f_{1}, \ldots, f_{n-1}\right) .
\end{aligned}
$$

The relation between the two coordinates comes from the following bijection of generators

$$
\left(g_{0}: g_{1}: \cdots: g_{n}\right) \longrightarrow\left(g_{0}^{-1} g_{1}, g_{1}^{-1} g_{2}, \ldots, g_{n-1}^{-1} g_{n}\right)
$$

which correctly transforms one definition of $\partial$ to the other. It follows that the two chain complexes are canonically isomorphic, and therefore by abuse of notation we denote either by $C_{*}(G)$, and write an element either in homogeneous or inhomogeneous coordinates as convenient.

Let $R$ be a commutative ring. The homology of the complex $C_{*}(G) \otimes R$ is denoted $H_{*}(G ; R)$, and the homology of the adjoint complex $\operatorname{Hom}\left(C_{*}(G), R\right)$ is denoted $H^{*}(G ; R)$. If $R=\mathbb{Z}$, we abbreviate these groups to $H_{*}(G)$ and $H^{*}(G)$ respectively. If $G$ is a topological group, and we want to stress that this is the abstract group (co)homology, we denote these groups by $H_{*}\left(G^{\delta}\right)$ and $H^{*}\left(G^{\delta}\right)$ respectively ( $\delta$ denotes the discrete topology).

We give a geometrical interpretation of this complex. The simplicial realization of the complex $C_{*}(G)$ is a model for the classifying space $B G$, where $G$ has the discrete topology. If $G$ is torsion-free, an equivalent model for $E G$ is the complete simplex on the elements of $G$. In this case, since $G$ is torsion-free, it acts freely and properly discontinuously on this simplex, with quotient $B G$. If we label vertices of $E G$ tautologically by elements of $G$, the labels on each simplex give homogeneous coordinates on the quotient. If we label edges of $E G$ by the difference of the labels on the vertices at the ends, then the labels are well-defined on the quotient; the labels on the $n$ edges between consecutive vertices of an $n$-simplex, with respect to a total order of the vertices, give inhomogeneous coordinates.

In particular, the cohomology $H^{*}(G)$ is just the cohomology of the $K(G, 1)$, that is, of the unique (up to homotopy) aspherical space with fundamental group isomorphic to $G$. If $G$ is not torsion-free, this equality of groups is nevertheless true.

The cohomology of the group $\mathrm{Homeo}^{+}\left(S^{1}\right)$ is known by a general theorem of Mather and Thurston (see [36] or [40] for details and more references): 
Theorem 2.3.2 (Mather, Thurston) For any manifold $M$, there is an isomorphism of cohomology rings

$$
H^{*}\left(\operatorname{Homeo}(M)^{\delta} ; \mathbb{Z}\right) \cong H^{*}(\text { BHomeo }(M) ; \mathbb{Z})
$$

where $B H o m e o(M)$ denotes the classifying space of the topological group of homeomorphisms of $M$, and the left hand side denotes the group cohomology of the abstract group of homeomorphisms of $M$.

For $M=S^{1}$ or $\mathbb{R}^{2}$, the topological group $\operatorname{Homeo}^{+}(M)$ is homotopy equivalent to a circle. For $S^{1}$, this is trivial. For $\mathbb{R}^{2}$, we observe that $\mathrm{Homeo}^{+}\left(\mathbb{R}^{2}\right)$ is the stabilizer of a point in $\mathrm{Homeo}^{+}\left(S^{2}\right)$, and then apply a theorem of Kneser [18] about the homotopy type of $\mathrm{Homeo}^{+}\left(S^{2}\right)$. It follows that $\mathrm{BHomeo}^{+}(M)$ in either case is homotopic to $\mathbb{C P}^{\infty}$, and therefore there is an isomorphism of rings

$$
H^{*}\left(\text { Homeo }^{+}\left(\mathbb{R}^{2}\right) ; \mathbb{Z}\right) \cong H^{*}\left(\text { Homeo }^{+}\left(S^{1}\right) ; \mathbb{Z}\right) \cong \mathbb{Z}[e]
$$

where $[e]$ is a free generator in degree 2 called the Euler class.

An algebraic characterization of the Euler class can be given.

Definition 2.3.3 For any group $G$ with $H^{1}(G ; \mathbb{Z})=1$, there is a universal central extension

$$
0 \longrightarrow A \longrightarrow \widehat{G} \longrightarrow G \longrightarrow 0
$$

where $A$ is abelian, with the property that for any other central extension

$$
0 \longrightarrow B \longrightarrow G^{\prime} \longrightarrow G \longrightarrow 0
$$

there is a unique homomorphism from $\widehat{G} \rightarrow G^{\prime}$, extending uniquely to a morphism of short exact sequences.

A non-split central extension $G^{\prime}$ can be characterized as the universal central extension of $G$ iff $G$ is perfect (i.e. $H^{1}(G ; \mathbb{Z})=1$ ) and every central extension of $G^{\prime}$ splits. See Milnor [24] for more details.

For $G=$ Homeo $^{+}\left(S^{1}\right)$, the universal central extension is denoted Homeo $\widetilde{H}^{+}\left(S^{1}\right)$, and can be identified with the preimage of $\mathrm{Homeo}^{+}\left(S^{1}\right)$ in $\mathrm{Homeo}^{+}(\mathbb{R})$ under the covering map $\mathbb{R} \rightarrow S^{1}$. The center of $\widetilde{\mathrm{Homeo}}^{+}\left(S^{1}\right)$ is $\mathbb{Z}$, and the class of this $\mathbb{Z}$ extension is called the Euler class. By the universal property of this extension, one sees that this class is the generator of $H^{2}\left(\operatorname{Homeo}^{+}\left(S^{1}\right) ; \mathbb{Z}\right)$. This can be summarized by a short exact sequence

$$
0 \longrightarrow \mathbb{Z} \longrightarrow \text { Homeo }^{+}\left(S^{1}\right) \longrightarrow \text { Homeo }^{+}\left(S^{1}\right) \longrightarrow 0 .
$$

The following construction is found in [38]. An equivalent construction is given in $[17]$. 
Construction 2.3.4 (Thurston) Let $G$ be a countable CO group, and let $\rho: G \rightarrow$ Homeo $^{+}\left(S^{1}\right)$ be constructed as in Theorem 2.2.14 so that the point $p$ has trivial stabilizer. For each triple $g_{0}, g_{1}, g_{2} \in G$ of distinct elements, define the cocycle

$$
c\left(g_{0}: g_{1}: g_{2}\right)=\left\{\begin{aligned}
1 & \text { if }\left(g_{0}(p), g_{1}(p), g_{2}(p)\right) \text { is positively oriented } \\
-1 & \text { otherwise. }
\end{aligned}\right.
$$

It is clear that $c$ is well-defined on the homogeneous coordinates for $C_{2}(G)$. Then extend $c$ to degenerate triples by setting it equal to 0 if at least two of its coefficients are equal.

The fact that the circular order on triples of points in $S^{1}$ is compatible on quadruples is exactly the condition that the coboundary of $c$ is 0 - that is, $c$ is a cocycle, and defines an element $[c] \in H^{2}(G ; \mathbb{Z})$.

The following (related) construction is found in [11]:

Construction 2.3.5 (Ghys) Let $G$ be a countable CO group. Let $\rho$ : $G \rightarrow$ Homeo $^{+}\left(S^{1}\right)$ be constructed as in Theorem 2.2.14. By abuse of notation, we identify $G$ with its image $\rho(G)$. Let $\widehat{G}$ denote the preimage of $G$ in the extension $\widetilde{H}^{+}{ }^{+}\left(S^{1}\right) \subset \mathrm{Homeo}^{+}(\mathbb{R})$. Define a section $s: G \rightarrow \widehat{G}$ uniquely by the property that $s(g)(0) \in[0,1)$. For each pair of elements $g_{0}, g_{1} \in G$, define the cocycle

$$
e\left(g_{0}, g_{1}\right)=s\left(g_{0} g_{1}\right)^{-1} s\left(g_{0}\right) s\left(g_{1}\right)(0) .
$$

Then one can check that $e$ is a cocycle on $C_{2}(G)$ in inhomogeneous coordinates, and defines an element $[e] \in H^{2}(G ; \mathbb{Z})$. Moreover, $e$ takes values in $\{0,1\}$.

The following lemma can be easily verified; for a proof, we refer to [38] or [17].

Lemma 2.3.6 (Ghys, Jekel, Thurston) Let $G$ be a countable circularlyordered group. The cocycles $e, c$ satisfy

$$
2[e]=[c] .
$$

Moreover, the class [e] is the Euler class of the circular order on $G$.

Actually, the restriction to countable groups is not really necessary. One can define the cocycles $c, e$ directly from a circular order on an arbitrary group $G$. This is actually done in [38] and [11]; we refer the reader to those papers for the more abstract construction. 
Theorem 2.3.7 Let $G$ be a circularly-ordered group with Euler class $[e] \in$ $H^{2}(G ; \mathbb{Z})$. If $[e]=0$, then $G$ is left-ordered. In any case, the central extension of $G$ corresponding to the class $[e]$ is left-orderable.

Proof We prove the theorem for $G$ countable; the general case is proved in [11].

From the definition of $s$ in Construction 2.3.5 and Lemma 2.3.6, we see that $e$ is the obstruction to finding some (possibly different) section $G \rightarrow \widehat{G}$. But $\widehat{G}$ is a subgroup of the group $\mathrm{Homeo}^{+}(\mathbb{R})$. Now, every finitely-generated subgroup of $\mathrm{Homeo}^{+}(\mathbb{R})$ is left-orderable, by Theorem 2.2.14. It follows by Lemma 2.1.6 that the entire group $\operatorname{Homeo}^{+}(\mathbb{R})$ is left-orderable; in particular, so is $\widehat{G}$.

\subsection{Bounded cohomology and the Milnor-Wood inequality}

Construction 2.3.4 and Construction 2.3.5 do more than give an explicit representative cocycle of the Euler class; they verify that this cocycle has a further additional property, namely that the Euler class is a bounded cocycle on $G$.

Definition 2.4.1 Suppose $R=\mathbb{R}$ or $\mathbb{Z}$. Define an $L_{1}$ norm on $C_{i}(G)$ in the obvious way by

$$
\left\|\sum_{j} s_{j}\left(g_{0}(j): g_{1}(j): \cdots: g_{i}(j)\right)\right\|_{1}=\sum_{j}\left|s_{j}\right| .
$$

Dually, the $L_{\infty}$ norm is partially defined on $\operatorname{Hom}\left(C_{i}(G) ; R\right)$, and the subspace consisting of homomorphisms of finite $L_{\infty}$ norm is denoted $\operatorname{Hom}_{b}\left(C_{i}(G) ; R\right)$. The coboundary takes cochains of finite $L_{\infty}$ norms to cocycles of finite $L_{\infty}$ norm, and therefore we can take the cohomology of the subcomplex. This cohomology is denoted $H_{b}^{*}(G ; \mathbb{R})$ and is called the bounded cohomology of $G$. For an element $\alpha \in H_{b}^{*}(G ; \mathbb{R})$, the norm of $\alpha$, denoted $\|\alpha\|_{\infty}$ or just $\|\alpha\|$, is the infimum of $\|c\|_{\infty}$ over cocycles $c$ with $[c]=\alpha$.

When we do not make coefficients explicit, the norm of a bounded cocycle refers to its norm amongst representatives with $\mathbb{R}$ coefficients.

In this language, the famous Milnor-Wood inequality [25], [42] can be expressed as follows:

Theorem 2.4.2 (Milnor-Wood) Let $G$ be a circularly-ordered group. Then the Euler class $[e]$ of $G$ is an element of $H_{b}^{2}(G)$ with norm $\|[e]\| \leq \frac{1}{2}$. 
Proof Let $e$ be the cocycle constructed by Ghys. Then $\frac{c}{2}=e-\frac{1}{2}$ is homologous to $e$, and has norm $\leq \frac{1}{2}$.

We will see in Section 4 that although $\mathrm{Homeo}^{+}\left(S^{1}\right)$ and $\mathrm{Homeo}^{+}\left(\mathbb{R}^{2}\right)$ have the same cohomology as abstract groups, their bounded cohomology groups are very different. This difference persists to the smooth category, as we shall see.

\section{$3 \quad$ Planar groups with bounded orbits}

The purpose of this section is to show that every group of $C^{1}$ orientationpreserving homeomorphisms of $\mathbb{R}^{2}$ with a bounded orbit is circularly-orderable. The main tools will be the Thurston stability theorem, and certain generalizations of the braid groups. In the course of the proof we also show that the mapping class group of a compact totally disconnected set in the plane is circularly-orderable, and the mapping class group rel. boundary of such a set in the disk is left-orderable. This generalizes a theorem of Dehornoy [7] on orderability of the usual (finitely-generated) braid groups.

\subsection{Prime ends}

In this section we describe some elements of the theory of prime ends. For details of proofs and references, consult [30] or [22].

Prime ends are a technical tool, developed in conformal analysis, to study the boundary behaviour of conformal maps which take the unit disk in $\mathbb{C}$ to the interior $U$ of a region $K$ whose boundary is not locally connected. They were introduced by Carathéodory in [6]. If $\partial K$ is locally connected, then the prime ends of $\partial K$ are just the proper homotopy classes of proper rays in $U$.

Definition 3.1.1 Let $U$ be a bounded open subset of $\mathbb{R}^{2}$, and let $K$ denote its closure. Fix the notation $\partial K=K \backslash U$. Notice that this might not be the frontier of $K$ in the usual sense. A null chain is a sequence of proper arcs $\left(C_{i}, \partial C_{i}\right) \subset(K, \partial K)$ where $\operatorname{int}\left(C_{i}\right) \subset U$ is an embedding, but whose endpoints are not necessarily distinct, such that the following conditions are satisfied:

(1) $\overline{C_{n}} \cap \overline{C_{n+1}}=\emptyset$

(2) $C_{n}$ separates $C_{0}$ from $C_{n+1}$ in $U$

(3) The diameter of the $C_{n}$ converges to 0 as $n \rightarrow \infty$. 
A prime end of $U$ is an equivalence class of null chains, where two null chains $\left\{C_{i}\right\},\left\{C_{i}^{\prime}\right\}$ are equivalent if for every sufficiently large $m$, there is an $n$ such that $C_{m}^{\prime}$ separates $C_{0}$ from $C_{n}$, and $C_{m}$ separates $C_{0}^{\prime}$ from $C_{n}^{\prime}$.

For such a set $U$, denote the set of prime ends of $U$ by $\mathscr{P}(U)$.

Example 3.1.2 Let $D$ denote the open unit disk in $\mathbb{C}$. Then $\mathscr{P}(D)$ is in natural bijective correspondence with $\partial \bar{D}$.

Notice that any homeomorphism of $\mathbb{R}^{2}$ which fixes $U$ as a set will induce an automorphism of $\mathscr{P}(U)$. On the other hand, a self-homeomorphism of $U$ which does not extend continuously to $\bar{U}$ will not typically induce an automorphism of $\mathscr{P}(U)$, since for instance the image of a properly embedded arc $C_{i}$ with endpoints on $\bar{U} \backslash U$ will not necessarily limit to well-defined endpoints in $U$.

Lemma 3.1.3 Let $U$ be a simply-connected, bounded, open subset of $\mathbb{R}^{2}$. Then the set of prime ends $\mathscr{P}(U)$ admits a natural circular order.

Proof Let $\varphi: U \rightarrow D$ be a uniformizing map. Then the set of prime ends of $U$ is taken bijectively to the set of prime ends of $D$. This is not entirely trivial; it is contained in proposition 2.14 and theorem 2.15 in [30]. In any case, this map identifies $\mathscr{P}(U)$ with $\partial \bar{D}$. Thus $\mathscr{P}(U)$ inherits a natural circular ordering from $\partial \bar{D}$. If $\varphi^{\prime}$ is another uniformizing map, then $\varphi^{\prime} \circ \varphi^{-1}$ is a Möbius transformation of $\partial \bar{D}$, and therefore preserves the circular order.

If $\varphi: U \rightarrow V$ is a conformal map between simply connected domains, then let $\varphi_{*}: \mathscr{P}(U) \rightarrow \mathscr{P}(V)$ denote the corresponding map between prime ends. As in the proof of Lemma 3.1.3, the proof that $\varphi_{*}$ is well-defined is found in [30].

Lemma 3.1.4 Suppose $\varphi: \mathbb{R}^{2} \rightarrow \mathbb{R}^{2}$ fixes $U$ as a set, and fixes the prime ends of $U$. Then $\varphi$ fixes $\bar{U} \backslash U$ pointwise.

Proof If $f: D \rightarrow U$ is a uniformizing map, then $f$ has a radial limit at $\zeta \in S^{1}$ iff the prime end $f_{*}(\zeta) \in \mathscr{P}(U)$ is accessible; that is, if there is a Jordan arc that lies in $U$ except for one endpoint, and that intersects all but finitely many crosscuts of some null-chain of $f_{*}(\zeta)$. The endpoints of this Jordan arc is called an accessible point. It is known (see [30]) that the set of accessible points is dense in $\bar{U} \backslash U$. But an automorphism which fixes all prime ends must fix all accessible points, and therefore must fix $\bar{U} \backslash U$ pointwise. 


\subsection{Groups which stabilize a point}

In this subsection we state the Thurston stability theorem, and from this deduce information about the group of $C^{1}$ homeomorphisms of $\mathbb{R}^{2}$ which stabilizes a point.

The Thurston stability theorem is proved in [37]. Since many people will only be familiar with the 1-dimensional version of this theorem, we indicate the idea of the proof.

Theorem 3.2.1 (Thurston stability theorem) Let $p$ be a point in a smooth manifold $M^{n}$. Let $G$ be a group of germs at $p$ of $C^{1}$ homeomorphisms of $M^{n}$ which fix $p$. Let $L: G \rightarrow G L(n, \mathbb{R})$ denote the natural homomorphism obtained by linearizing $G$ at p. Let $L(G)$ denote the image of $G$, and $K(G)$ the kernel of $L$. Then $K(G)$ is locally indicable.

Proof The idea of the proof is as follows. Let $H<K(G)$ be a finitely generated subgroup, with generators $h_{1}, \ldots h_{m}$. Let $p_{i} \rightarrow p$ be some convergent sequence. If we rescale the action near $p_{i}$ so that every $h_{j}$ moves points a bounded distance, but some $h_{k}(i)$ moves points distance 1 , then the rescaled actions vary in a precompact family. It follows that we can extract a limiting nontrivial action, which by construction will be an action by translations. In particular, $H$ is indicable, and $K(G)$ is locally indicable.

Now we make this more precise. Change coordinates so that $p$ is at the origin. Then each generator $h_{i}$ can be expressed in local coordinates as a sum

$$
h_{i}(x)=x+y\left(h_{i}\right)(x)
$$

where $\left|y\left(h_{i}\right)(x)\right|=o(x)$ and satisfies $\left.y\left(h_{i}\right)^{\prime}\right|_{0}=0$. For each $\epsilon>0$, let $U_{\epsilon}$ be an open neighborhood of 0 on which $\left|y\left(h_{i}\right)^{\prime}\right|<\epsilon$ and $\left|y\left(h_{i}\right)(x)\right|<|x| \epsilon$. Now, for two indices $i, j$ the composition has the form

$$
\begin{aligned}
h_{i} \circ h_{j}(x) & =x+y\left(h_{j}\right)(x)+y\left(h_{i}\right)\left(x+y\left(h_{j}\right)(x)\right) \\
& =x+y\left(h_{j}\right)(x)+y\left(h_{i}\right)(x)+O\left(\epsilon y\left(h_{j}\right)(x)\right) .
\end{aligned}
$$

In particular, the composition deviates from $x+y\left(h_{i}\right)(x)+y\left(h_{j}\right)(x)$ by a term which is small compared to $\max \left(y\left(h_{i}\right)(x), y\left(h_{j}\right)(x)\right)$.

Now, choose some sequence of points $x_{i} \rightarrow 0$. For each $i$ define the map $v_{i}: H \rightarrow \mathbb{R}^{n}$ where $v_{i}(h)=y(h)\left(x_{i}\right)$. Let $w_{i}=\sup _{j \leq m}\left|v_{i}\left(h_{j}\right)\right|$, and define $v_{i}^{\prime}(h)=v_{i} / w_{i}$. It follows that the functions $v_{i}^{\prime}$ are uniformly bounded on each $h \in H$, and therefore there is some convergent subsequence. Moreover, by the 
estimate above, on this subsequence, the maps $v_{i}^{\prime}$ converge to a homomorphism $v^{\prime}: H \rightarrow \mathbb{R}^{n}$. On the other hand, by construction, there is some index $j$ such that $\left|v_{i}^{\prime}\left(h_{j}\right)\right|=1$. In particular, the homomorphism $v^{\prime}$ is nontrivial, and $H$ surjects onto a nontrivial free abelian group, and we are done.

Lemma 3.2.2 Let $G$ be a group of germs of orientation preserving $C^{1}$ homeomorphisms of $\mathbb{R}^{2}$ at a fixed point $p$. Then $G$ is circularly-orderable. Moreover, if the image $L(G)$ of $G$ in $G L^{+}(2, \mathbb{R})$ obtained by linearizing the action at $p$ is left-orderable, then $G$ itself is left-orderable.

Proof By the Thurston stability theorem, if $L: G \rightarrow L(G)$ denotes the homomorphism onto the linear part of $L(G)$, then the kernel $K(G)$ is locally indicable, and therefore by Theorem 2.1.11, $K(G)$ is LO. That is, we have a short exact sequence

$$
0 \longrightarrow K(G) \longrightarrow G \longrightarrow L(G) \longrightarrow 0
$$

where $K(G)$ is LO. If the image $L(G)$ is LO, then so is $G$ by Lemma 2.1.9.

Moreover, since $G$ is orientation preserving, $L(G)<\mathrm{GL}^{+}(2, \mathbb{R})$ where $\mathrm{GL}^{+}$ denotes the subgroup of GL with positive determinant. There is a homomorphism from $\mathrm{GL}^{+}(2, \mathbb{R})$ to $\mathrm{SL}(2, \mathbb{R})$ with kernel $\mathbb{R}^{+}$. We write this as a short exact sequence:

$$
0 \longrightarrow \mathbb{R}^{+} \longrightarrow \mathrm{GL}^{+}(2, \mathbb{R}) \longrightarrow \mathrm{SL}(1, \mathbb{R}) \longrightarrow 0 .
$$

The group $\operatorname{SL}(2, \mathbb{R})$ double covers $\operatorname{PSL}(2, \mathbb{R})$, and can be thought of as the group of orientation-preserving transformations of the connected double cover of $\mathbb{R} \mathbb{P}^{1}$ which are the pullback of projective transformations of $\mathbb{R P}^{1}$ by $\operatorname{PSL}(2, \mathbb{R})$. In particular, $\mathrm{SL}(2, \mathbb{R})$ is a subgroup of $\mathrm{Homeo}^{+}\left(S^{1}\right)$, and is therefore CO. It follows by Lemma 2.2.12 that $\mathrm{GL}^{+}(2, \mathbb{R})$ is $\mathrm{CO}$, and therefore so is $L(G)$.

By another application of Lemma 2.2.12, $G$ is CO.

\subsection{Groups which stabilize one or more compact regions}

The main point of this subsection is to prove Theorem 3.3.6, which says that a group of $C^{1}$ orientation-preserving homeomorphisms of the plane which fixes a compact set $K$ with connected complement is circularly-orderable, and a group which fixes at least two such sets is left-orderable.

Now, suppose that a group $G$ stabilizes the disjoint compact connected sets $K_{1}, K_{2}, \ldots$. For each $K_{i}$, exactly one complementary component is unbounded. 
So without loss of generality, we can fill in these bounded complementary regions and assume $G$ stabilizes disjoint compact connected sets $K_{1}, \ldots$ with connected complement for all $i$.

Remark 3.3.1 A compact set $K_{i}$ which is an absolute neighborhood retract satisfies the hypotheses of Alexander duality, and has connected complement in $\mathbb{R}^{2}$ iff $H_{1}\left(K_{i} ; \mathbb{Z}\right)=0$. However, the well-known example of the "topologist's circle" shows that in general, the vanishing of homology is not sufficient to show that the complement is connected.

We show how each region $K_{i}$ gives rise to a $\mathrm{CO}$ group $G_{i}$ which is naturally a quotient of $G$.

Construction 3.3.2 If $K_{i}$ consists of more that one point, the complement of $K_{i}$ in the sphere $S^{2}=\mathbb{R}^{2} \cup \infty$ is conformally a disk, and we let $G_{i}$ denote the image of $G$ in $\operatorname{Aut}\left(\mathscr{P}\left(S^{2} \backslash K_{i}\right)\right)$. Note that $G_{i}$ is CO, by Lemma 3.1.3.

If $K_{i}$ consists of a single point and $G$ acts $C^{1}$ near $K_{i}$, let $G_{i}$ denote the germ of $G$ at $K_{i}$. By Lemma 3.2.2, $G_{i}$ is CO, and is actually LO if the linear part $L(G)$ of $G$ at $K_{i}$ is LO.

Remark 3.3.3 Notice that there is a natural circular order on $G_{i}$ in the first case, but not in the second. However, in the second case, the Euler class of the circular order provided by Lemma 3.2.2 is just the circular Euler class of the linear part $L(G)$ of $G$ at $K_{i}$, acting projectively on the unit tangent bundle, and is therefore natural.

In particular, the set $K_{i}$ gives a homomorphism from $G$ to a product

$$
G \longrightarrow \prod_{i} G_{i}
$$

of $\mathrm{CO}$ groups. The product of any number of left-orderable groups is leftorderable. This follows immediately from Lemma 2.1.9 and Lemma 2.1.6. But a product of $\mathrm{CO}$ groups is not necessarily $\mathrm{CO}$. In this section we will show that in the context above, if $G$ stabilizes two disjoint compact connected sets $K_{1}, K_{2}$ with connected complement, then the groups $G_{1}, G_{2}$ are actually both LO.

Lemma 3.3.4 Let $G$ act by $C^{1}$ orientation-preserving homeomorphisms on the plane. Suppose that $G$ stabilizes two disjoint compact connected sets $K_{1}, K_{2}$ with connected complement. Then the groups $G_{1}, G_{2}$ provided by Construction 3.3.2 are circularly-orderable, and the pullback of their Euler classes to $G$ are zero. 
Proof First suppose $K_{1}$ contains at least two points.

For a compact, connected set $K_{1} \subset \mathbb{R}^{2}$ with connected complement, the complement of $\mathbb{R}^{2} \backslash K_{1}$ is homeomorphic to an annulus $A_{1}$. Let $\widetilde{A_{1}}$ denote the universal cover of $A_{1}$, and denote by $\widehat{G_{1}}$ the central extension of $G_{1}$

$$
0 \longrightarrow \mathbb{Z} \longrightarrow \widehat{G_{1}} \longrightarrow G_{1} \longrightarrow 0 \text {. }
$$

This requires some explanation. The Riemann surface $\widetilde{A_{1}}$ is noncompact, and topologically is an open disk. Under a uniformizing map, there is a unique point $p \in S^{1}$ such that every proper ray $r$ in $\widetilde{A_{1}}$ which projects to an unbounded proper ray in $A_{1}$ is asymptotic to $p$. If we cut $S^{1}$ at $p$, we get a copy of $\mathbb{R}$, which by abuse of notation we refer to as the set of prime ends $\mathscr{P}\left(\widetilde{A_{1}}\right)$

Since by hypothesis $K_{1}$ consists of more than one point, there is a natural map $\mathscr{P}\left(\widetilde{A_{1}}\right) \rightarrow \mathscr{P}\left(S^{2} \backslash K_{1}\right)$ which is just a covering map $\mathbb{R} \rightarrow S^{1}$ under the identification of $\mathscr{P}\left(S^{2} \backslash K_{1}\right)$ with $\mathscr{P}(D)=S^{1}$ by a uniformizing map. Then the group $\widehat{G_{1}}$ is just the usual preimage of a subgroup of $\operatorname{Homeo}^{+}\left(S^{1}\right)$ in Homeo ${ }^{+}\left(S^{1}\right)$.

In particular, the class of this extension is the Euler class of the circular ordering on $G_{i}$. In order to show that the pullback of this Euler class to $G$ is trivial, it suffices to show that the restriction homomorphism res: $G \rightarrow \operatorname{Homeo}^{+}\left(A_{1}\right)$ lifts to the covering space $\widehat{\mathrm{res}}: G \rightarrow \operatorname{Homeo}^{+}\left(\widetilde{A_{1}}\right)$.

Let $\widehat{K_{2}}$ be a lift of $K_{2}$ to $\widetilde{A_{1}}$. Then for each element $g \in G$, there is a unique lift $\widehat{\text { res }}: g \rightarrow$ Homeo $^{+}\left(\widetilde{A_{1}}\right)$ which stabilizes $\widehat{K_{2}}$. By uniqueness, this defines the desired section.

The case that $K_{1}$ consists of a single point is very similar. The complement $\mathbb{R}^{2} \backslash K_{1}$ is again an annulus, and there is $\mathrm{A}$ covering $\widetilde{A_{1}} \rightarrow A_{1}$. There is a central extension

$$
0 \longrightarrow \mathbb{Z} \longrightarrow \widehat{G_{1}} \longrightarrow G_{1} \longrightarrow 0
$$

where $G_{1}$ is the germ of $G$ at $K_{1}$, and $\widehat{G_{1}}$ is the corresponding germ of the preimage of $G$ in Homeo ${ }^{+}\left(\widetilde{A_{1}}\right)$. Again, choosing a lift $\widehat{K_{2}}$ of $K_{2}$ to $\widetilde{A_{1}}$ determines a unique section $G \rightarrow \operatorname{Homeo}^{+}\left(\widetilde{A_{1}}\right)$ whose germ is $\widehat{G_{1}}$. So the pullback of the Euler class to $G$ is trivial in this case too.

We now establish a technical lemma about groups which act in a $C^{1}$ fashion and have indiscrete fixed point set.

Lemma 3.3.5 Suppose $G$ acts faithfully by $C^{1}$ orientation-preserving homeomorphisms of the plane, and suppose that the fixed point set $\operatorname{fix}(G)$ is not discrete. Then $G$ is left-orderable. 
Proof Let $p \in \operatorname{fix}(G)$ in the frontier of $\operatorname{fix}(G)$ be a limit point of distinct $p_{i} \in \operatorname{fix}(G)$. Then the $p_{i}$ contain a subsequence which approach $p$ radially along some vector $v$. It follows that the linearization of $G$ at $p$, denoted $L(G)$, fixes the vector $v$, and therefore the image is contained in some conjugate of the affine group of the line $\mathrm{Aff}^{+}(\mathbb{R})$. The affine group of the line is an extension of $\mathbb{R}$ by $\mathbb{R}$ :

$$
0 \longrightarrow \mathbb{R} \longrightarrow \mathrm{Aff}^{+}(\mathbb{R}) \longrightarrow \mathbb{R} \longrightarrow 0
$$

where the homomorphism to $\mathbb{R}$ is given by the $\log$ of the dilation, and the kernel is the subgroup of translations. It follows that $\mathrm{Aff}^{+}(\mathbb{R})$ is left-orderable by Lemma 2.1.9.

It follows from Lemma 3.2.2 that the germ of $G$ at $p$ is LO. In particular, if $H$ is any finitely-generated subgroup of $G$, then we can apply this reasoning to a limit point in the frontier of $\operatorname{fix}(H)$ and see that the germ of $H$ at this limit point is left-orderable. In particular, $H$ is LO-surjective. Since $H$ was arbitrary, by Theorem 2.1.11 the group $G$ is LO as required.

We now have all the tools to prove the main result of this subsection:

Theorem 3.3.6 Let $G$ act faithfully by $C^{1}$ orientation-preserving homeomorphisms of the plane. Suppose that $G$ stabilizes disjoint compact connected sets $\left\{K_{i}\right\}$ such that each $K_{i}$ has connected complement. Then $G$ is $C O$. If there is more than one region $K_{i}$, then $G$ is $L O$.

Proof By Lemma 3.3.4 there is a homomorphism

$$
G \longrightarrow G_{i}
$$

for each $i$, where the image group is $\mathrm{CO}$, and the pullback of the Euler class of this circular ordering to $G$ is trivial if there is more than one region $K_{i}$.

Let $K$ denote the kernel of this homomorphism. For each region $K_{i}$ with more than one point, Lemma 3.1.4 implies that $K$ fixes $\partial K_{i}$ pointwise. For each region $K_{i}$ consisting of a single point, every element of $K$ fixes some neighborhood of $K_{i}$ pointwise. It follows that for each finitely-generated subgroup $H$ of $K$ the set of fixed points of $H$ is not isolated. In particular, by Lemma 3.3.5, every finitely-generated subgroup of $K$ is LO, and therefore $K$ is LO by Lemma 2.1.6.

By Lemma 2.2.12, $G$ is CO. Moreover, the Euler class of this circular ordering is the pullback of the Euler class of $G_{i}$ under the homomorphism $G \rightarrow G_{i}$. In particular, if there is more than one region $K_{i}$, the Euler class of the circular ordering on $G$ is zero, and therefore $G$ is LO, as claimed. 


\subsection{Totally disconnected planar sets}

If $G$ acts faithfully on $\mathbb{R}^{2}$ and merely permutes a collection of disjoint compact regions $\left\{K_{i}\right\}$ we can study the image of $G$ in the group of homotopy classes of all orientation preserving homeomorphisms of $\mathbb{R}^{2}$ which permute the $\left\{K_{i}\right\}$. We will call such a group a generalized braid group. The following construction is a convenient simplification.

Construction 3.4.1 Suppose $G$ acts faithfully on $\mathbb{R}^{2}$ and preserves some bounded set $K$. Take the closure of $K$, fill in bounded complementary regions, and label the components of the result as $\left\{K_{i}\right\}$.

Since the $K_{i}$ are obtained naturally from $K, G$ permutes the components $\left\{K_{i}\right\}$. Observe that the union of the $K_{i}$ is compact, and that the $K_{i}$ are disjoint, compact, and have connected complement. Then we can define an equivalence relation on $\mathbb{R}^{2}$ by quotienting each $K_{i}$ to a point.

Definition 3.4.2 A decomposition $\mathscr{G}$ of a space is a partition into compact subsets. A decomposition is upper semi-continuous if for every decomposition element $\zeta \in \mathscr{G}$ and every open set $U$ with $\zeta \subset U$, there exists an open set $V \subset U$ with $\zeta \subset V$ such that every $\zeta^{\prime} \in \mathscr{G}$ with $\zeta^{\prime} \cap V \neq \emptyset$ has $\zeta^{\prime} \subset U$. The decomposition is monotone if its elements are connected.

It is clear that the equivalence relation in Construction 3.4.1 is monotone and upper semi-continuous.

The following theorem is proved by R. L. Moore in [27]:

Theorem 3.4.3 (Moore) Let $\mathscr{G}$ be a monotone upper semi-continuous decomposition of a topological sphere $S$ such that no decomposition element separates $S$. Then the quotient space obtained by collapsing each decomposition element to a point is a topological sphere.

Definition 3.4.4 Suppose $G$ acts on a space $X$ and preserves a monotone equivalence relation $\sim$. Then the action of $G$ descends to an action on $X / \sim$. We say the two actions are monotone equivalent.

Lemma 3.4.5 Let $G$ act on $\mathbb{R}^{2}$ in such a way as to permute a union of disjoint compact regions. Then this action is monotone equivalent to an action which leaves invariant a compact, totally disconnected, $G$-invariant subset. 
Proof The fact that $\mathbb{R}^{2} / \sim$ is homeomorphic to $\mathbb{R}^{2}$ follows from Moore's Theorem 3.4.3. Since Construction 3.4.1 is natural, the equivalence relation it defines is $G$-invariant, and therefore the action of $G$ descends to the quotient. It is clear from the construction that $C$ is totally disconnected.

Since $G$ acts on $\mathbb{R}^{2}$ and permutes $C$, we can study a minimal subset of $C$; that is, a closed invariant nonempty subset of $\mathbb{R}^{2}$ which is minimal with respect to these properties. Amongst all invariant subsets, a minimal subset $C$ can be characterized by the property that every $G$ orbit contained in $C$ is dense in $C$. Such a subset will be either finite or perfect. Here, a set is perfect if every point is a limit point.

The Moore-Kline theorem gives a useful "normal form" for totally disconnected compact planar sets. This theorem is proved in [28]:

Theorem 3.4.6 (Moore, Kline) Let $C$ be a totally disconnected compact set in $\mathbb{R}^{2}$. Then there is a homeomorphism of $\mathbb{R}^{2}$ to itself which takes $C$ to a closed subset of the arc $[0,1]$ contained in the $x$-axis.

We will use this theorem in the next subsection. Note that this theorem implies that a perfect, totally disconnected subset of $\mathbb{R}^{2}$ is a tame Cantor set.

\subsection{Generalized braid groups}

Definition 3.5.1 For a surface $S$ (possibly with boundary $\partial S$ ) the relative orientation-preserving mapping class group, denoted $\mathrm{MCG}^{+}(S, \partial S)$, is the group of homotopy classes of orientation-preserving homeomorphisms of $S$ to itself which are fixed on $\partial S$. We generally just call this the mapping class group for short.

Let $C$ be a compact, totally disconnected subset of the open unit disk $D$. Then the generalized braid group of $C$, denoted $B_{C}$, is the relative mapping class group $\mathrm{MCG}^{+}(\bar{D} \backslash C, \partial \bar{D})$ and the planar generalized braid group of $C$, denoted $B_{C}^{\prime}$, is the mapping class group $\mathrm{MCG}^{+}\left(\mathbb{R}^{2} \backslash C\right)$.

By extending a homeomorphism of $D$ fixed on $\partial \bar{D}$ by the identity, we see that there is a natural homomorphism $B_{C} \rightarrow B_{C}^{\prime}$. This gives rise to a central extension

$$
0 \longrightarrow \mathbb{Z} \longrightarrow B_{C} \longrightarrow B_{C}^{\prime} \longrightarrow 0
$$


where the generator of $\mathbb{Z}$ is the (positive) Dehn twist of $D^{2}$ along a boundary parallel loop.

If $C$ is a finite set with $n$ points, then $B_{n}$ is the usual braid group, and $B_{n}^{\prime}$ is the usual quotient of $B_{n}$ by its center. A reference for the theory of braid groups, with particular reference to questions of orderability or circular orderability, is $[8]$.

We come to the first main result of this paper.

The proof of this theorem follows the same general approach as Thurston's proof of the orderability of the usual (finitely-generated) braid groups [32], but several technical complications arise because the surfaces $D^{2} \backslash C$ and $\mathbb{R}^{2} \backslash C$ are not of finite type.

Theorem A Let $C$ be a compact, totally disconnected subset of the open unit disk $D$. Then $B_{C}^{\prime}$ is circularly-orderable, and $B_{C}$ is left-orderable.

Proof If $C$ consists of a single point, both groups are trivial. If $C$ consists of two points, $B_{C}^{\prime}=\mathbb{Z} / 2 \mathbb{Z}$ and $B_{C}=\mathbb{Z}$, so the theorem is satisfied in that case. So without loss of generality we assume that $C$ contains at least 3 points.

First we show that $B_{C}^{\prime}$ is circularly-orderable. Let $S=S^{2} \backslash C$. Then $S$ is a hyperbolic surface, and we can identify the universal cover $\widetilde{S}=\mathbb{H}^{2}$. We work in the unit disk model of $\mathbb{H}^{2}$, so that $\widetilde{S}$ can be compactified by adding the circle at infinity $S_{\infty}^{1}$. There is a short exact sequence

$$
\left.0 \longrightarrow \pi_{1}(S) \longrightarrow \widehat{\operatorname{MCG}(S}\right) \longrightarrow \operatorname{MCG}(S) \longrightarrow 0 .
$$

Here $\widehat{\mathrm{MCG}(S)}$ denotes the group of homotopy classes of homeomorphisms of $\widetilde{S}$ which commute with the action of $\pi_{1}(S)$, and cover homeomorphisms of $S$.

It turns out that there is a naturally defined injective homomorphism $\sigma$ from the mapping class group $\operatorname{MCG}\left(\mathbb{R}^{2} \backslash C\right)$ to $\left.\widehat{\operatorname{MCG}(S}\right)$. We define $\sigma$ as follows. Let $[\phi] \in \operatorname{MCG}\left(\mathbb{R}^{2} \backslash C\right)$ denote a typical element, and let $\phi$ be a representative. Then $\phi$ extends continuously to a homeomorphism $\phi_{S}$ of $S$ which fixes the point $\infty$. Choose a lift $\widehat{\infty}$ of $\infty$ in $\widetilde{S}$. Then there is a unique lift of $\phi_{S}$ to $\widetilde{S}$ which fixes $\widehat{\infty}$; call this lift $\phi_{\widetilde{S}}$. Then define $\sigma$ by

$$
\sigma([\phi])=\left[\phi_{\widetilde{S}}\right] .
$$

Since the construction of $\phi_{\widetilde{S}}$ depends continuously on $\phi$, it follows that the class $\left[\phi_{\widetilde{S}}\right]$ is well-defined. 
We show that it is injective. If $\left[\phi_{\widetilde{S}}\right]=\left[\psi_{\widetilde{S}}\right]$ for some $\phi, \psi$ then there is a homotopy $\Psi_{\widetilde{S}}: \widetilde{S} \times I \rightarrow \widetilde{S}$ with $\Psi_{\widetilde{S}}(\cdot, 0)=\psi_{\widetilde{S}}$ and $\Psi_{\widetilde{S}}(\cdot, 1)=\phi_{\widetilde{S}}$ which at every point is $\pi_{1}(S)$ equivariant. It follows that $\Psi_{\widetilde{S}}$ descends to a map $\Psi_{S}: S \times I \rightarrow S$ which is a homotopy between $\psi_{S}$ and $\phi_{S}$. Moreover, under the track of this homotopy, the point $\infty$ moves in a homotopically inessential loop, since by construction, this loop lifts to $\widetilde{S}$. So we can find another homotopy $\Psi_{S}^{\prime}$, homotopic to $\Psi_{S}$, which fixes $\infty$. It follows that $\Psi_{S}^{\prime}$ restricts to $\Psi: \mathbb{R}^{2} \backslash C \times I \rightarrow$ $\mathbb{R}^{2} \backslash C$ which is a homotopy from $\psi$ to $\phi$. This proves that the map is an injection.

In fact, it is not hard to show that the map $\sigma$ is actually an isomorphism, but this is unnecessary for our purposes.

We now show that the group $\widehat{\operatorname{MCG}(S)}$ is circular. First of all we construct an action of $\widehat{\operatorname{MCG}(S)}$ on a circularly-ordered set.

The conformal structure that $S$ inherits as an open submanifold of $\mathbb{R}^{2}$ determines a natural complete hyperbolic structure on $S$. This gives an identification of the universal cover $\widetilde{S}$ with the hyperbolic plane $\mathbb{H}^{2}$, which can be compactified by its ideal boundary which is a circle $S_{\infty}^{1}$. An orientation on $S$ determines one on $\widetilde{S}$ and therefore also on $S_{\infty}^{1}$. Since the hyperbolic structure on $S$ is complete, the limit set $\Lambda$ of $\pi_{1}(S)$ is the entire circle $S_{\infty}^{1}$. A dense subset of $S_{\infty}^{1}$ consists of endpoints of axes of hyperbolic translations $\alpha \in \pi_{1}(S)$. Notice that since $\pi_{1}(S)$ is discrete, the stabilizer of a point in $\pi_{1}(S)$ is cyclic, so distinct axes have distinct endpoints. Such endpoints are in bijective correspondence with the set $\mathscr{E}$ whose elements are maximal hyperbolic cyclic subgroups $\langle\alpha\rangle$ of $\pi_{1}(S)$ together with a choice of generator for $H_{1}(\langle\alpha\rangle ; \mathbb{Z})$. It follows that $\mathscr{E}$ inherits a circular order by its bijection with a subset of the circle $S_{\infty}^{1}$.

The group $\widehat{\operatorname{MCG}(S)}$ induces an automorphism of $\pi_{1}(S)$ by conjugation, and therefore induces an action on the set $\mathscr{E}$. We verify that this action preserves the cyclic order.

To see this, let $\alpha, \beta$ be hyperbolic elements of $\pi_{1}(S)$ with axes $l_{\alpha}, l_{\beta}$. Let $[\phi] \in$ $\widehat{\operatorname{MCG}(S)}$, and let $\phi$ be a representative. The images of the axes $\phi\left(l_{\alpha}\right), \phi\left(l_{\beta}\right)$ are periodic under the elements $[\phi]_{*}(\alpha)=[\phi] \alpha[\phi]^{-1}$ and $[\phi]_{*}(\beta)=[\phi] \beta[\phi]^{-1}$ in $\pi_{1}(S)$ respectively, so they converge to well-defined end points in $S_{\infty}^{1}$, which are exactly the endpoints of the axes of $l_{[\phi]_{*}(\alpha)}, l_{[\phi]_{*}(\beta)}$. Moreover, they are either disjoint or cross transversely in exactly one point, according to whether $l_{\alpha}, l_{\beta}$ are disjoint or cross transversely in exactly one point respectively. It follows that $l_{\alpha}, l_{\beta}$ cross iff $l_{[\phi]_{*}(\alpha)}, l_{[\phi]_{*}(\beta)}$ cross. See Figure 1. 

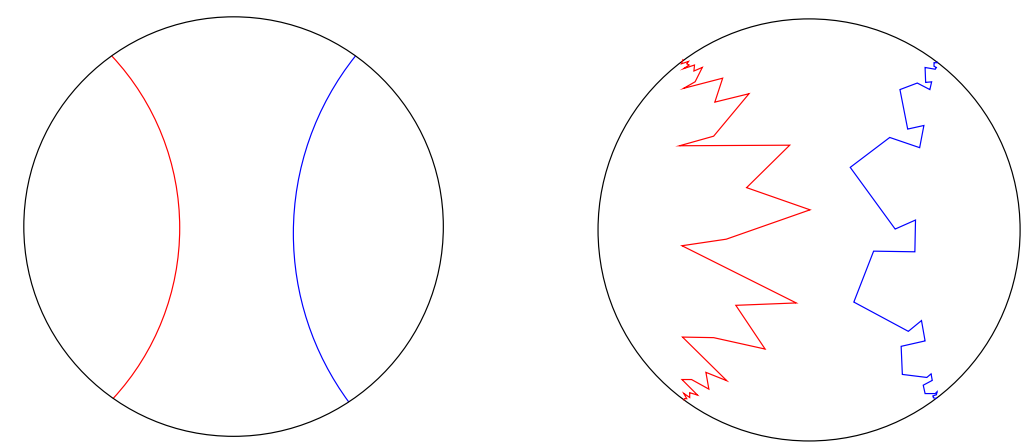

Figure 1: The axes $l_{\alpha}, l_{\beta}$ are disjoint iff their images under $\phi$ are disjoint. But this is true iff the axes $l_{[\phi]_{*}(\alpha)}, l_{[\phi]_{*}(\beta)}$ are disjoint. This defines a natural action of $\widehat{\operatorname{MCG}(S)}$ on the circularly-ordered set of endpoints of axes.

The circular ordering on the endpoints of $l_{\alpha}, l_{\beta}$ is determined by this crossing information, the orientation of the axes, and if the axes do not intersect, the relationship between the orientation of the axes and the orientation of the subsurface of $\widetilde{S}$ that they both cobound. It follows that the circular order of the endpoints of the $l_{\alpha}, l_{\beta}$ is taken to the circular order of the endpoints of the axes $l_{[\phi]_{*}(\alpha)}, l_{[\phi]_{*}(\beta)}$ under $[\phi]$, and therefore the action of $\widehat{\operatorname{MCG}(S)}$ preserves the circular order on $\mathscr{E}$.

It remains to show this action is faithful. One way to do this is to find a maximal collection of pairwise disjoint geodesic loops $\Gamma=\left\{\gamma_{i}\right\}$ in $S$. We want to be slightly careful about the choice of $\Gamma$; we want it to be maximal, and also closed as a subset of $S$. In particular, the geodesics $\gamma_{i}$ should not accumulate. We show how to choose such a collection.

By the Moore-Kline Theorem 3.4.6 we can assume, after a homeomorphism of $\mathbb{R}^{2}$, that the set $C$ is contained as a closed, totally disconnected subset of a horizontal arc $I$ in the $x$-axis. In particular, the complement $I \backslash C$ consists of a countable collection of open intervals $I_{i}$. For each connected complementary open interval $I_{i}$ we let $p_{i}$ denote the midpoint. We inductively produce a maximal set of round circles $S_{k}$ which are symmetric in the $x$-axis, and intersect the $x$ axis in points $p_{i}, p_{j}$ subject to the constraint that the circles are nonintersecting, and the $k$ th circle is chosen to be the biggest circle with diameter $\leq 2^{-k}$. Note that a maximal collection of such circles has the property that each complementary domain contains at most finitely many unmatched $p_{i}$.

Our initial guess for $\Gamma$ is the set of geodesics isotopic to $S_{i}$. Note that some $S_{i}$ might be boundary parallel; we throw these out and just look at the ones with 
geodesic representatives. This is not quite maximal, but each complementary region is of finite type, and there are only finitely many complementary regions whose diameter (in the Euclidean metric in $\mathbb{R}^{2}$ ) is $>\epsilon$ for any $\epsilon$. So we just take a maximal (finite) collection of geodesic loops in each such complementary region, and add these loops to $\Gamma$. Notice that this union of geodesic loops is closed, doesn't accumulate anywhere (in $S$ ) and is maximal.

Now, by hypothesis, $[\phi]$ preserves every hyperbolic cyclic subgroup $\langle\alpha\rangle$ of $\pi_{1}(S)$; in particular, the projection $\phi_{S}$ of a representative $\phi$ to $S$ preserves every oriented free homotopy class of loop which is not boundary parallel. So the collection $\Gamma$ is taken to a collection of curves which are curvewise isotopic to $\Gamma$. By straightening these geodesics inductively, we can homotope $\phi_{S}$ to $\phi_{S}^{\prime}$ which fixes $\Gamma$ pointwise. Since $\Gamma$ was maximal, every complementary surface to $\Gamma$ is either a thrice punctured sphere, a twice punctured disk, a once punctured annulus, or a pair of pants. An automorphism of one of these subsurfaces which preserves every hyperbolic cyclic subgroup is isotopic to the identity, so $\phi_{S}$ is actually isotopic to the identity, and therefore $[\phi]$ is in $\pi_{1}(S)$. Now, an element of $\pi_{1}(S)$ which, under conjugation, takes a hyperbolic cyclic subgroup to itself in an orientation preserving way must actually commute with that subgroup. But this contradicts the fact that $\pi_{1}(S)$ is nonelementary.

The conclusion is that $\widehat{\mathrm{MCG}(S)}$ acts faithfully in an order preserving way on a circularly-ordered set, and therefore $\widehat{\operatorname{MCG}(S)}$ is circularly-ordered, by Theorem 2.2.14. Since $\sigma: B_{C}^{\prime} \rightarrow \widehat{\operatorname{MCG}(S)}$ is injective, $B_{C}^{\prime}$ is circularly-ordered too.

Proving that $B_{C}$ is left-orderable is similar; let $p \in \mathbb{R}^{2} \backslash D$ be some point. Define $S$ to be obtained from $\mathbb{R}^{2} \backslash(C \cup p)$ by adding a point to compactify $\mathbb{R}^{2}$, and then removing $(C \cup p)$. Exactly as in the previous case, there is an injective homomorphism from $B_{C}$ to $\widehat{\widehat{M C G}(S)}$, and an invariant circular order on $\widehat{\operatorname{MCG}(S)}$. Moreover, the image of $B_{C}$ stabilizes the parabolic subgroup corresponding to a small loop around $p$, since representatives of $B_{C}$ act trivially there. In particular, the image of $B_{C}$ stabilizes a point in $S_{\infty}^{1}$, and therefore it is left-orderable, as required.

\subsection{Groups with bounded orbits}

We are now in a position to prove the following:

Theorem B Let $G$ be a group of orientation preserving $C^{1}$ homeomorphisms of $\mathbb{R}^{2}$ with a bounded orbit. Then $G$ is circularly-orderable. 
Proof Let $p$ be a point with a bounded orbit, and consider the closure of the orbit of $p$. This is a compact union of disjoint compact components which is permuted by $G$.

We apply Construction 3.4.1 to fill in the sets with disconnected complement to get a new collection of connected sets $\left\{K_{i}\right\}$ which are compact with connected complements, whose union is compact, and whose components are permuted by $G$. We then apply Lemma 3.4 .5 to get a monotone equivalent action of $G$ on $\mathbb{R}^{2}$ which leaves invariant a totally disconnected compact set $C$.

By theorem A, the image of $G$ in the mapping class group of $\mathbb{R}^{2} \backslash C$ is circularlyorderable. Denote the kernel by $K$. Then $K$ fixes $C$ pointwise, and therefore with respect to the original action, $K$ preserves the components $\left\{K_{i}\right\}$ componentwise.

By Theorem 3.3.6, $K$ is circularly-orderable, and is left-orderable if there are at least two components of $K_{i}$. If there is only one component $K_{i}$, the quotient $C$ is a single point, and the mapping class group of $\mathbb{R}^{2} \backslash C$ is trivial, in which case $G=K$. So either $G$ is circularly-orderable, or else it maps to a circularlyorderable group with left-orderable kernel. By Lemma 2.2.12, $G$ is circularlyorderable, as claimed.

\section{The Euler class and smoothness}

The purpose of this section is to give a complete classification of which Euler classes can arise for orientation-preserving actions of oriented surface groups on $\mathbb{R}^{2}$ in every degree of smoothness.

One main result is that the Euler class of the group of $C^{\infty}$ orientation-preserving diffeomorphisms of the plane is an unbounded class, in stark contrast with the case of Homeo ${ }^{+}\left(S^{1}\right)$ and the Milnor-Wood inequality. In particular, if $S$ is a closed surface of genus at least 2 , there is a $C^{\infty}$ action of $\pi_{1}(S)$ on the plane with arbitrary Euler class. The other main result is that the genus one case is more rigid: we show that an orientation-preserving $C^{1}$ action of $\mathbb{Z} \oplus \mathbb{Z}$ on the plane has vanishing Euler class.

Using these facts, we prove the existence of a finitely-generated torsion-free group of homeomorphisms of the plane which is not circularly-orderable. 


\subsection{The Euler class and winding number}

We give two descriptions of the Euler class of a group $G$ acting on $\mathbb{R}^{2}$ by a representation $\rho: G \rightarrow$ Homeo $^{+}\left(\mathbb{R}^{2}\right)$. The first description is more algebraic.

Construction 4.1.1 Let $G_{\infty}$ denote the germ of $G$ at $\infty$. Let $A$ be a punctured disk neighborhood of $\infty$, and let $\widetilde{A}$ be the universal cover of $A$. Then there is a central extension

$$
0 \longrightarrow \mathbb{Z} \longrightarrow \widehat{G}_{\infty} \longrightarrow G_{\infty} \longrightarrow 0
$$

where $\widehat{G}_{\infty}$ denotes the subgroup of periodic germs of homeomorphisms of $\widetilde{A}$. The class of this extension pulls back by the natural restriction homomorphism $G \rightarrow G_{\infty}$ to give the Euler class $\rho^{*}[e] \in H^{2}(G ; \mathbb{Z})$.

To see that this is the Euler class, observe that it defines a non-split extension, and therefore determines a non-trivial element of $H^{2}\left(\mathrm{Homeo}^{+}\left(\mathbb{R}^{2}\right) ; \mathbb{Z}\right)$. By evaluating this class on some suitable homology cycles (e.g. Example 4.2.1 in the sequel) one sees that it is a primitive element, and therefore it is equal to the generator of $H^{2}\left(\mathrm{Homeo}^{+}\left(\mathbb{R}^{2}\right) ; \mathbb{Z}\right)$, as claimed.

The next description is geometric, in terms of a winding number. Notice that for this definition to make sense, we must assume the action is at least $C^{1}$.

Construction 4.1.2 Now we give a more geometric construction of the Euler class, in terms of how it pairs with elements of $H_{2}(G ; \mathbb{Z})$. Assume the action is at least $C^{1}$. It is a fact that for any group $G$, an integral class $\sigma \in H_{2}(G ; \mathbb{Z})$ can be realized as the image of a map from a closed orientable surface $S$ of genus $\geq 1$ into a $K(G, 1)$

$$
f: S \longrightarrow K(G, 1) .
$$

Given a representation $\rho$, we get an induced action

$$
\rho: \pi_{1}(S) \longrightarrow \text { Homeo }^{+}\left(\mathbb{R}^{2}\right) .
$$

Let $g$ be the genus of $S$, and suppose that we obtain $S$ by gluing the sides of a $4 g$-sided polygon $P_{g}$ in pairs in the usual way. Let $d_{\rho}: \widetilde{S} \rightarrow \mathbb{R}^{2}$ be an equivariant $C^{1}$ map, called a developing map and look at the restriction

$$
d_{\rho}: \partial P_{g} \longrightarrow \mathbb{R}^{2}
$$

where $P_{g}$ now denotes a fundamental domain, by abuse of notation. If necessary, we can assume that $d_{\rho}$ is an immersion on edges of $\partial P_{g}$. 
Let $\delta: S^{1} \rightarrow \mathbb{R}^{2}$ be obtained from $d_{\rho}$ by smoothing the corners at each vertex of $\partial P_{g}$. Then the Euler class of $\rho$, evaluated on $[S]$, can be calculated by the formula

$$
\rho^{*}([e])([S])=\operatorname{index}(\delta)+1-2 g
$$

where index $(\delta)$ denotes the usual winding number of the tangent vector $T \delta$ around $S^{1}$. See Figure 2 for an example of a hypothetical action of $\mathbb{Z} \oplus \mathbb{Z}$ with Euler number 1 . In the figure, $\alpha, \beta$ denote generators of $\mathbb{Z} \oplus \mathbb{Z}$.

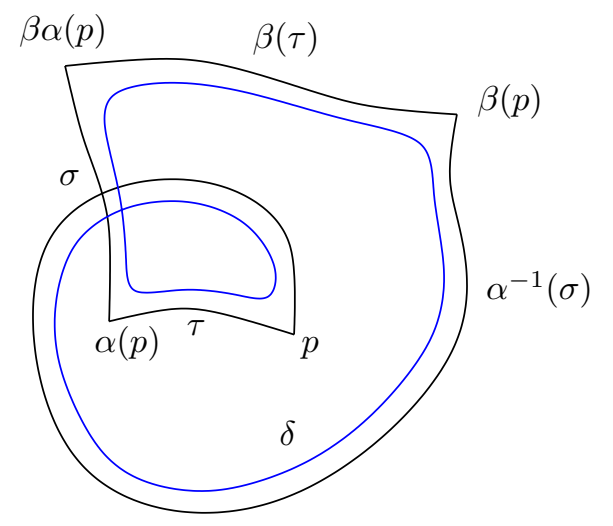

Figure 2: This hypothetical action of $\mathbb{Z} \oplus \mathbb{Z}$ satisfies $\operatorname{index}(\delta)=2$, so $\rho^{*}([e])([\Sigma])=1$.

In practice we would like to work with developing maps which are as degenerate as possible, in order to reduce the combinatorial complexity of the calculation. So to calculate the "correct" smoothing along a degenerate edge, we must perturb the picture near a degenerate edge in an equivariant way; i.e. the perturbations must be combinatorially equivalent along degenerate edges which are paired by the edge pairing translations.

The justification for this formula is as follows. Associated to a representation $\rho: G \rightarrow$ Homeo $^{+}\left(\mathbb{R}^{2}\right)$ there is an associated foliated $\mathbb{R}^{2}$ bundle $E_{\rho}$ over $B G$. Here $B G$ is the classifying space for $G$ as a discrete group; that is, it is a $K(G, 1)$. It is defined as the quotient of the product $\mathbb{R}^{2} \times E G$ by the action of $G$, where $E G$ is the universal cover of $B G$, and

$$
\gamma(p, q)=(\rho(\gamma)(p), \gamma(q))
$$

By basic obstruction theory, the Euler class $\rho^{*}([e])$ is the obstruction to trivializing $E_{\rho}$ as an $\mathbb{R}^{2}$ bundle over the 2 -skeleton of $B G$. See for example [16] for details.

If $\Sigma$ is a closed, orientable surface of genus $g$, and $G=\pi_{1}(\Sigma)$, then $B G=\Sigma$. Since $\mathbb{R}^{2}$ is contractible, we can always find a section $\sigma: \Sigma \rightarrow E_{\rho}$ of $E_{\rho}$ over $\Sigma$. 
Suppose $\rho$ is $C^{1}$. Then $E_{\rho}$ is naturally a $C^{1}$ manifold, and we can choose $\sigma$ to be smooth. The pullback of the unit tangent bundle in the fiber direction by $\sigma$ defines an orientable circle bundle $E_{\rho}^{\prime}$ over $\Sigma$. A trivialization of $E_{\rho}^{\prime}$ defines a trivialization of $E_{\rho}$ by exponentiation, and conversely, a (smooth) trivialization of $E_{\rho}$ defines a trivialization of $E_{\rho}$ by restriction. Since $\operatorname{Diffeo}^{+}\left(\mathbb{R}^{2}\right)$ and Homeo $^{+}\left(\mathbb{R}^{2}\right)$ are homotopic as topological groups, $E_{\rho}$ can be trivialized iff it can be smoothly trivialized.

It follows that the Euler class of $\rho$ is the Euler class of the circle bundle $E_{\rho}^{\prime}$. The section $\sigma$ defines a developing map $d_{\rho}$. If necessary, perturb $d_{\rho}$ to be an immersion on each edge of $P_{g}$. This immersion gives a trivialization of $E_{\rho}^{\prime}$ along the pushoff (into $P_{g}$ ) of each edge of $\partial P_{g}$. This trivialization on either side of an edge of the 1-skeleton disagrees along the edge, and therefore the trivialization can be extended across each edge of the 1-skeleton of $S$ with a single saddle singularity, and across the vertex with a single sink singularity. This section extends non-singularly over a fundamental domain $P_{g}$ iff the index of the smoothed curve $d_{\rho}(\delta)$ is 0 . It follows that the Euler class, evaluated on $\Sigma$, is $\operatorname{index}(\delta)+1-2 g$. The formula follows.

See Figure 3 for a picture of the trivialization in a neighborhood of a typical edge and vertex in the genus 2 case.
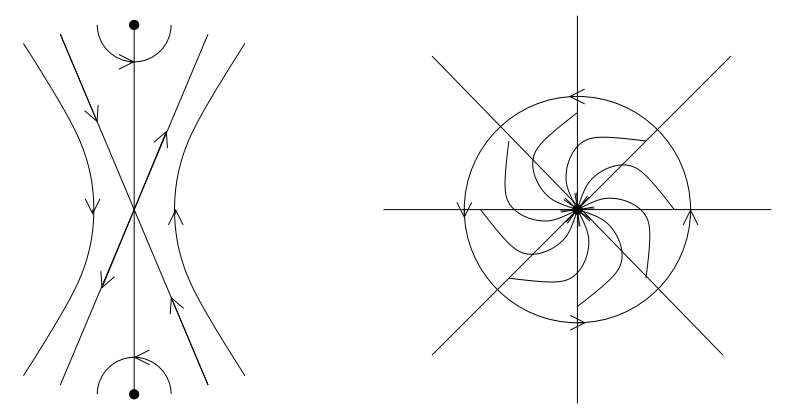

Figure 3: The trivialization can be extended over each edge with a saddle singularity, and over the vertex with a sink singularity.

\subsection{Unboundedness of the Euler class}

In this subsection we construct $C^{\infty}$ actions on the plane of $\pi_{1}(S)$, where $S$ is the closed surface of genus 2, with arbitrary Euler class.

We begin with the following example, due to Bestvina: 
Example 4.2.1 (Bestvina) Let $C_{i}$ be the round circle in $\mathbb{R}^{2}$ centered at the origin, with radius $2^{i}$, for $i \in \mathbb{Z}$. That is, the $C_{i}$ are a bi-infinite nested sequence of circles about 0 . Let $\alpha$ be the composition of a positive Dehn twist in each circle $C_{i}$.

More precisely, let $A_{i}$ be the annulus whose boundary components are the circle of radius $0.99 \times 2^{i}$ and the circle of radius $1.01 \times 2^{i}$. Let $\psi:[0,1] \rightarrow[0,1]$ be a smooth homeomorphism, infinitely tangent to a constant map at 0 and 1 . Then let $\alpha$ be the identity outside the disjoint union of the $A_{i}$, and let its restriction to the circle of radius $(0.99+t \times 0.02) \times 2^{i}$ be a rotation through angle $2 \pi \psi(t)$ for $t \in[0,1]$. In particular, note that $\alpha$ is smooth away from the origin.

Let $\beta$ be the dilation centered at 0 sending $z \rightarrow 2 z$. Then the commutator $[\alpha, \beta]=\mathrm{Id}$, and it is easy to see that

$$
\langle\alpha, \beta\rangle \cong \mathbb{Z} \oplus \mathbb{Z}
$$

On the other hand, the image of the boundary of the polygon $\partial P_{1}$ under $d_{\rho}$ from Construction 4.1.2 is isotopic to the configuration in Figure 2. In particular, the Euler number of this $\mathbb{Z} \oplus \mathbb{Z}$ action is 1 .

If $G_{i}$ is a subgroup of $\mathbb{Z} \oplus \mathbb{Z}$ of index $i$, then $G_{i}$ is also isomorphic to $\mathbb{Z} \oplus \mathbb{Z}$, and the induced action has Euler number $i$. In particular, the Euler class $[e] \in H^{2}\left(\right.$ Homeo $\left.^{+}\left(\mathbb{R}^{2}\right) ; \mathbb{Z}\right)$ is unbounded.

The action of $\mathbb{Z} \oplus \mathbb{Z}$ is not $C^{1}$ at the origin. Bestvina posed the question of whether the Euler class was unbounded for $C^{1}$ actions. We show that the answer to this question is positive, and in fact show that the Euler class is unbounded for $C^{\infty}$ actions.

Theorem $\mathbf{C}$ For each integer $i$, there is a $C^{\infty}$ action

$$
\rho_{i}: \pi_{1}(S) \rightarrow \text { Diffeo }^{+}\left(\mathbb{R}^{2}\right)
$$

where $S$ denotes the closed surface of genus 2, satisfying

$$
\rho_{i}^{*}([e])([S])=i .
$$

In particular, the Euler class $[e] \in H^{2}\left(\right.$ Diffeo $\left.^{+}\left(\mathbb{R}^{2}\right) ; \mathbb{Z}\right)$ is unbounded.

Proof As in Example 4.2.1, let $C_{j}$ be the round circle in $\mathbb{R}^{2}$ with radius $2^{j}$ centered at the origin. Let $\alpha$ be the composition of a positive Dehn twist in each circle $C_{j}$ for $j \geq 0$, and let $\beta$ be the dilation centered at 0 sending $z \rightarrow 2 z$. Then $\alpha, \beta$ are both $C^{\infty}$, and the commutator $\left[\alpha^{i}, \beta\right]$ is the $i$ th power 
of a positive Dehn twist in the circle $C_{0}$. Now let $D_{j}$ be the round circle in $\mathbb{R}^{2}$ with radius 1 centered at the point with $x$ coordinate $3 j$ and $y$ coordinate 0 . Note that $C_{0}=D_{0}$. Let $\gamma$ be the composition of a positive Dehn twist in each circle $D_{j}$ for $j \geq 0$, and let $\delta$ be the translation parallel to the $x$-axis through distance 3 . Then $\gamma, \delta$ are both $C^{\infty}$, and the commutator $\left[\gamma^{i}, \delta\right]$ is the $i$ th power of a positive Dehn twist in the circle $D_{0}$. It follows that the there is an identity

$$
\left[\alpha^{i}, \beta\right]=\left[\gamma^{i}, \delta\right]
$$

and the group generated by $\alpha^{i}, \beta, \gamma^{i}, \delta$ is a quotient of $\pi_{1}(S)$ for $S$ the closed surface of genus 2 .

We calculate the Euler class of this action. One method is to find a polygon $P_{2}$ and a developing map $d_{\rho}: \partial P_{2} \rightarrow \mathbb{R}^{2}$ which is smoothed to $\delta: \partial P_{2} \rightarrow \mathbb{R}^{2}$ with $\operatorname{index}(\delta)=3+i$. It follows by Construction 4.1.2 that

$$
\rho_{i}^{*}([e])([S])=3+i+1-2 g=i .
$$

This is left as a pleasant exercise for the reader.

Alternatively, we can use Construction 4.1.1 to evaluate the Euler class. Since the commutators $\left[\alpha^{i}, \beta\right]$ and $\left[\gamma^{i}, \delta\right]$ have compact support, their images in the group of germs at infinity are trivial. It follows that in the group of germs, the genus 2 surface group breaks up into two genus 1 surface groups. The first $\mathbb{Z} \oplus \mathbb{Z}$ has Euler class $i$, as in Bestvina's example. The group generated by $\gamma^{i}$ and $\delta$ fixes the negative $x$-axis. It follows that we can find a section of the universal central extension by choosing a lift which fixes a lift of the germ at infinity of the negative $x$-axis. In particular, the extension splits, and the Euler class on the second $\mathbb{Z} \oplus \mathbb{Z}$ is trivial.

Theorem $\mathrm{C}$ raises the following natural question:

Question 4.2.2 Is the Euler class a bounded class on the group of real analytic orientation-preserving diffeomorphisms of $\mathbb{R}^{2}$ ?

Some weak evidence for a positive answer to this question is the following:

Theorem 4.2.3 The group $\operatorname{Poly}^{+}\left(\mathbb{R}^{2}\right)$ of polynomial orientation-preserving homeomorphisms is circularly-orderable.

Proof Let $r_{0}$ be the positive real axis, and let $R$ denote the set of germs at infinity of translates of $r_{0}$ by orientation-preserving polynomial homeomorphisms. Observe that $R$ contains the germs at infinity of every straight ray. It 
turns out that $R$ is a naturally circularly-ordered set. To see this, observe that if $\alpha: \mathbb{R}^{2} \rightarrow \mathbb{R}^{2}$ is a polynomial homeomorphism, then $\alpha\left(r_{0}\right)$ is real algebraic, and therefore its germ at infinity is either equal to the germ at infinity of $r_{0}$, or is disjoint from it. If $\left\{r_{i}\right\}$ are a finite collection of disjoint, properly embedded rays in $\mathbb{R}^{2}$, then they are naturally circularly-ordered as follows. Let $D$ be a large closed disk whose boundary intersects the $r_{i}$ transversely. For each $r_{i}$, the intersection $r_{i} \cap \partial D$ is a finite set. This set inherits a natural ordering from the orientation on $r_{i}$. For each $i$, let $p_{i}$ be the greatest element of this set. Then the rays $r_{i}$ are circularly-ordered by the circular ordering of the $p_{i}$ in $\partial D$. One can verify that this ordering is independent of the choice of (sufficiently large) $D$, and therefore defines a circular ordering on the germs at infinity of the $r_{i}$.

Now, a polynomial homeomorphism of $\mathbb{R}^{2}$ which preserves the germ at infinity of a straight line must preserve the entire line as a set. Moreover, any homeomorphism of $\mathbb{R}^{2}$ which preserves every straight line as a set must be the identity. It follows that the natural homomorphism $\mathrm{Poly}^{+}\left(\mathbb{R}^{2}\right) \rightarrow \operatorname{Aut}(R)$ is injective. By Theorem 2.2.15, Poly ${ }^{+}\left(\mathbb{R}^{2}\right)$ is circularly-orderable.

\subsection{Torsion-free groups which are not left-orderable}

In this section we construct groups of orientation-preserving homeomorphisms of the plane which are not left-orderable. Of course, there are some very simple examples of such groups: any finite cyclic group acts on the plane by rotations, but the only finite left-orderable group is the trivial group. Benson Farb asked whether there are any examples of torsion-free groups which act on the plane but are not left or circularly-orderable. In this section, we construct an explicit example of such a group which is not left-orderable. In the next section, we show how to promote this example to show the existence of a finitely presented torsion-free subgroup of Homeo ${ }^{+}\left(\mathbb{R}^{2}\right)$ which is not circularly-orderable.

Theorem 4.3.1 There is a finitely presented torsion-free $C^{\infty}$ subgroup of Diffeo $^{+}\left(\mathbb{R}^{2}\right)$ which is not left-orderable.

Proof Let $G$ be given by the following presentation:

$$
G=\left\langle a, b, c, t \mid a^{2}=t, b^{3}=t, c^{7}=t, a b c=t^{3}\right\rangle .
$$

Then $G$ is the fundamental group of the Seifert fibered space

$$
M=(O o 0 \mid 3 ;(2,1),(3,1),(7,1))
$$


in the notation of Montesinos [26]. In particular, this is a 3-manifold with $\widehat{S L(2, \mathbb{R})}$ geometry, so $\widetilde{M}$ is homeomorphic to $\mathbb{R}^{3}$, and $G$ is torsion-free.

On the other hand, $\pi_{1}(M)$ is not left-orderable, and in fact admits no nontrivial homomorphism to $\mathrm{Homeo}^{+}(\mathbb{R})$. A more general fact is proved in [31], but it is easy enough to give a direct proof in our case. Suppose $G$ is left-orderable, so that we can disjointly decompose $G=P \cup N \cup \operatorname{Id}$ with $P \cdot P \subset P$ and $P^{-1}=N$, by Lemma 2.1.2. Without loss of generality, we can assume $t \in P$. Then since $t$ is a nontrivial positive power of each of the generators $a, b, c$ we must have $a, b, c \in P$ and also $a, b, c<t$. But then $a b c<t^{3}$ contradicting the fourth relation.

On the other hand, since $t$ is central, we can form the quotient $\Delta$ of $G$ by $\langle t\rangle$ with the presentation

$$
\Delta=\left\langle a, b, c \mid a^{2}=b^{3}=c^{7}=a b c=\mathrm{Id}\right\rangle
$$

so that there is a short exact sequence

$$
0 \rightarrow \mathbb{Z} \rightarrow G \rightarrow \Delta \rightarrow 0
$$

Now, the group $\Delta$ acts faithfully and discretely on $\mathbb{R}^{2}$ by $C^{\infty}$ homeomorphisms. To see this, observe e.g. by van Kampen's theorem that $\Delta$ is the fundamental group of the hyperbolic $(2,3,7)$ triangle orbifold, and therefore admits a (unique up to conjugacy and orientation) discrete faithful representation into $\operatorname{PSL}(2, \mathbb{R})=\operatorname{Isom}^{+}\left(\mathbb{H}^{2}\right)$. So after choosing a diffeomorphism of $\mathbb{H}^{2} \rightarrow \mathbb{R}^{2}$, we obtain a representation $\rho: \Delta \rightarrow \operatorname{Diffeo}^{+}\left(\mathbb{R}^{2}\right)$. We will construct a monotone equivalent (smooth) faithful action of $G$. Since $\rho(\Delta)$ is discrete, and the set of points with nontrivial stabilizer in $\Delta$ are discrete, we let $O$ be the orbit of a point with trivial stabilizer in $\Delta$, and choose a diffeomorphism of $\mathbb{R}^{2} \backslash O$ with $\mathbb{R}^{2} \backslash X$ where $X$ is the union of infinitely many nowhere accumulating disjoint closed disks. Let $\rho^{\prime}: G \rightarrow$ Diffeo $^{+}\left(\mathbb{R}^{2} \backslash X\right)$ be the pushforward of the representation $\rho$ under this diffeomorphism. We extend this action over $X$ in the following way. Since the components of $X$ are permuted by $\Delta$ with trivial stabilizer, we can identify $X=\Delta \times D$ where $D$ denotes the closed unit disk. Now let the kernel $\langle t\rangle$ of $\rho$ act on $D$ by some smooth diffeomorphism which is fixed in a collar neighborhood of the boundary and has infinite order, and take the product action on $\Delta \times D$. This product action is smooth, and since $t$ acts trivially on $\mathbb{R}^{2} \backslash X$, it is smooth on all of $\mathbb{R}^{2}$. This defines an extension of $\rho^{\prime}$ to all of $\mathbb{R}^{2}$ which is smooth and faithful.

Remark 4.3.2 In [31], it is actually proved that every action of the fundamental group of an exceptional Seifert fibered space $M$ on $\mathbb{R}$ is equivalent to 
the action of $\pi_{1}(M)$ on the space of leaves of some taut foliation of $M$ transverse to the circle fibration. By the Milnor-Wood inequality, such a foliation can only exist if the Euler class of the bundle is smaller in absolute value than the Euler characteristic of the base orbifold, which is a sphere with three exceptional fibers. In particular, for any such base orbifold, there are infinitely many Seifert fibered spaces, but only finitely many whose fundamental groups are left-orderable. On the other hand, by more or less repeating the construction in Theorem 4.3.1, one can see that the fundamental group of every Seifert fibered 3-manifold with infinite fundamental group admits a faithful $C^{\infty}$ representation in Diffeo ${ }^{+}\left(\mathbb{R}^{2}\right)$. Every such group is torsion-free. On the other hand, all of these groups are circularly-orderable, and contain finite index subgroups which are left-orderable.

\subsection{Torsion-free groups which are not circularly-orderable}

In this subsection we show that we can combine theorem $\mathrm{C}$ with the example from Theorem 4.3.1 to give an example of a torsion-free subgroup of Homeo $^{+}\left(\mathbb{R}^{2}\right)$ which is not circularly-orderable, thereby giving a complete positive answer to Farb's question. At one point, we make use of a powerful theorem of Tsuboi ([39]) about the homology of the group of $C^{1}$ homeomorphisms of $\mathbb{R}^{n}$, but aside from this, our construction is basically elementary.

The use of Tsuboi's theorem is not logically essential; one could avoid it by an explicit construction. But such a construction would add considerably to the length of the example, without being any more enlightening.

Our proof follows the model of an argument due to Ghys [13] which shows that there exist planar groups which are not circularly-orderable, using Example 4.2.1, and the fact that Homeo ${ }^{+}\left(\mathbb{R}^{2}\right)$ contains torsion elements.

First we prove a couple of lemmas.

Lemma 4.4.1 Let $G$ be the group from Theorem 4.3.1. Then if $\rho: G \rightarrow$ Homeo $^{+}\left(S^{1}\right)$ is a homomorphism for which $\rho(t)$ has a fixed point, $\rho$ is monotone equivalent to an action which factors through $\Delta=G /\langle t\rangle$. Moreover, $\rho$ is either trivial, or is semi-conjugate to the "standard" hyperbolic action coming from a faithful representation of $\Delta$ in $\operatorname{PSL}(2, \mathbb{R})$.

Proof Let $T \subset S^{1}$ be the fixed point set of $\rho(t)$. By blowing up the orbit of some $p \in T$, we can assume that $T$ has nonempty interior. Since $t$ is central, the subset $T$ is permuted by $\rho(G)$, so we can blow down $S^{1} \backslash T$ to get a monotone 
equivalent action on which $t$ acts trivially, so up to monotone equivalence, the action factors through $\Delta=G /\langle t\rangle$, as claimed.

Now, it is well-known that every homomorphism of $\Delta$ to Homeo ${ }^{+}\left(S^{1}\right)$ is either trivial, or monotone equivalent to a hyperbolic action. See [5] for a proof, which makes use of a theorem of Matsumoto [23].

Lemma 4.4.2 Let Diffeo $o_{c}^{+}\left(\mathbb{R}^{2}\right)$ denote the group of diffeomorphisms of $\mathbb{R}^{2}$ with compact support. There is a finitely-generated subgroup $L$ of $\operatorname{Diffeo}_{c}^{+}\left(\mathbb{R}^{2}\right)$ containing an element $t$ which is both a commutator in $L$, and conjugate to its square in $L$.

Proof Such subgroups are very easy to find. For example, let $\phi: \mathbb{R}^{2} \rightarrow D^{2}$ be a diffeomorphism such that $\left\|\phi^{\prime}\right\|$ decays very fast at infinity, and let $t$ be the pushforward by $\phi$ of a translation. Then $t$ is certainly both a commutator, and conjugate to its square. To see that $t$ is a commutator, observe that the commutator of two dilations with distinct centers is a translation. Moreover, a dilation with dilation constant 2 conjugates a translation to its square. If $\left\|\phi^{\prime}\right\|$ decays sufficiently quickly (for instance, exponential decay is sufficient) the pushforward of any element of $L$ to $D^{2}$ is $C^{\infty}$ tangent to the identity along $\partial D^{2}$.

With these lemmas proved, we can now give a positive answer to Farb's question.

Theorem 4.4.3 There exists a finitely-generated, torsion-free subgroup of Homeo ${ }^{+}\left(\mathbb{R}^{2}\right)$ which is not circularly-orderable.

Proof Let $G$ be the example from Theorem 4.3.1. By abuse of notation, we will actually think of $G$ as a subgroup of $\operatorname{Diffeo}^{+}\left(\mathbb{R}^{2}\right)$. We use the same notation for the generators of $G$. By construction, the support of $t$ is a nonaccumulating union of closed disks $\Delta \times D$. Moreover, the action of $t$ on $1 \times D$ can be chosen to be arbitrary, and the action on each other translate $g \times D$ is conjugate to the action on $1 \times D$. By Lemma 4.4.2, we can insist that the restriction of $t$ to $1 \times D$ has the following properties:

- There are diffeomorphisms $r, s$ whose commutator satisfies $[r, s]=t$.

- There is a diffeomorphism $q$ such that $q t q^{-1}=t^{2}$. 
Moreover, we can choose $q, r, s$ to have support equal to $\Delta \times D$, and further we can insist that the action on each translate $g \times D$ is conjugate to the action on $1 \times D$. Let $L$ denote the group generated by $q, r, s, t$. Note that $L$ is abstractly isomorphic to the group of the same name constructed in Lemma 4.4.2. Define

$$
G^{\prime}=\langle G, L\rangle
$$

Then by construction, $G^{\prime}$ maps surjectively onto $\Delta$ with kernel $L$, and is torsion-free.

Now, if $\rho^{\prime}: G^{\prime} \rightarrow$ Homeo $^{+}\left(S^{1}\right)$ is a homomorphism, then $\rho^{\prime}(t)$ is conjugate in Homeo $^{+}\left(S^{1}\right)$ to $\rho^{\prime}\left(t^{2}\right)$. In particular, the rotation number of $\rho^{\prime}(t)$ satisfies

$$
\operatorname{rot}\left(\rho^{\prime}(t)\right)=\operatorname{rot}\left(\rho^{\prime}\left(t^{2}\right)\right)=2 \operatorname{rot}\left(\rho^{\prime}(t)\right)
$$

and therefore this rotation number is equal to 0 , and $\rho^{\prime}(t)$ has a fixed point. For the definition and properties of rotation number, see [5].

It follows by Lemma 4.4.1 that up to monotone equivalence, $\left.\rho^{\prime}\right|_{G}$ is equivalent to the standard action of the fundamental group of the $(2,3,7)$ hyperbolic triangle orbifold on its circle at infinity.

The group $G$ contains a subgroup $H$ of index 168 corresponding to a congruence cover of the $(2,3,7)$ hyperbolic triangle orbifold. See for instance [19] for details. Since $G$ is a $\mathbb{Z}$ extension of the orbifold group, the subgroup $H$ is a $\mathbb{Z}$ extension of the fundamental group of a closed surface $\Sigma$ of genus 3. By passing to covers in the base direction, for arbitrarily large $m$, we can find a subgroup $H_{m}$ of $H$ of index $m$ which is a $\mathbb{Z}$ extension of the fundamental group of a closed surface $\Sigma_{m}$ of genus $2 m+1$. The Euler class of the extension corresponding to $H$ evaluated on the fundamental class of the genus 3 surface is some number $a$, and therefore the Euler number of the extension corresponding to $H_{m}$ is $m a$.

So there are elements

$$
\alpha_{1, m}, \beta_{1, m}, \ldots, \alpha_{2 m+1, m}, \beta_{2 m+1, m} \in G
$$

which map to standard generators of a surface group of genus $2 m+1$ under the homomorphism to $\Delta$, such that

$$
\prod_{i}\left[\alpha_{i, m}, \beta_{i, m}\right]=t^{m a} .
$$

Now, $\left[q^{n} r q^{-n}, q^{n} s q^{-n}\right]=t^{2^{n}}$, so for each $m$, we can express $t^{m a}$ as a product of approximately $\log m a$ commutators of this form. Together with the $\alpha_{i, m}, \beta_{i, m}$, we can produce surface subgroups $S_{m}$ of $G^{\prime}$ of genus approximately $2 m+$ 
$2 \log m a$. We know that the action of $\alpha_{i, m}, \beta_{i, m}$ on $S^{1}$ is "standard", up to semi-conjugacy. So by the Milnor-Wood inequality Theorem 2.4.2 and the fact that the restriction of $\rho^{\prime}$ to $H_{m}$ is standard, we can estimate the circular Euler class

$$
\left(\rho^{\prime}\right)^{*}([e])\left[S_{m}\right] \leq-4 m+4 \log m a
$$

which is as negative as we like for big $m$. Here the $-4 m$ term comes from the standard action of $H_{m}$, and the $4 \log m a$ term comes from Milnor-Wood. Moreover, since $r, s$ have support contained in the union of the disks $\Delta \times D$, the formula from Construction 4.1.2 implies that the planar Euler class of $S_{m}$ is $-4 m$, since we can choose a fundamental domain to calculate the Euler class which does not intersect the support of $r, s$.

Now, by a theorem of Tsuboi, the homology of the group of $C^{1}$ orientationpreserving diffeomorphisms of $\mathbb{R}^{2}$ is equal to $\mathbb{Z}$ in dimension 2 ; see [40] and [39]. Let $F_{2}$ denote the fundamental group of a surface of genus 2 . Let $\rho_{-4 m}$ be the representation constructed in theorem $\mathrm{C}$ with $i=-4 m$. Then $\rho_{-4 m}\left(F_{2}\right)$ and $S_{m}$ are homologous as subgroups of the group of $C^{1}$ orientationpreserving homeomorphisms of $\mathbb{R}^{2}$. By vanishing of the oriented cobordism group in dimension 3, we can take this homology to be an oriented 3-manifold. That is, there is a 3 -manifold $M$ with two boundary components, $\partial(M)=$ $\partial_{1}(M) \cup \partial_{2}(M)$, where $\partial_{1}(M)$ has genus 2 and $\partial_{2}(M)$ has genus approximately $2 m+2 \log m a$, and a $C^{1}$ representation $\sigma_{M}: \pi_{1}(M) \rightarrow \operatorname{Homeo}^{+}\left(\mathbb{R}^{2}\right)$ where $\left.\sigma_{M}\right|_{\partial_{1}(M)}$ is conjugate to $\left.\rho_{-4 m}\right|_{F_{2}}$, and $\left.\sigma_{M}\right|_{\partial_{2}(M)}$ is conjugate to the action of $S_{m}$. In fact, $C^{1}$ is superfluous for our application: it suffices that each element of $\sigma_{M}\left(\pi_{1}(M)\right)$ is $C^{1}$ on an open dense subset. Such a homology can be constructed by more elementary methods, since the boundary representation is $C^{\infty}$.

We can find an oriented irreducible $3-$ manifold $N$ with torsion-free fundamental group, and a degree 1 map $\varphi: N \rightarrow M$ which is a homeomorphism on the boundary. Then $\sigma_{M}$ induces a $C^{1}$ representation $\sigma_{N}: \pi_{1}(N) \rightarrow \mathrm{Homeo}^{+}\left(\mathbb{R}^{2}\right)$ with the same boundary behaviour. It follows that $\sigma_{N}$ extends to a $C^{1}$ action of the amalgamated free product

$$
G^{\prime \prime}=G^{\prime} *_{S_{m}} \pi_{1}(N)
$$

which is faithful on the subgroup $G^{\prime}$. Let $\sigma: G^{\prime \prime} \rightarrow$ Homeo $^{+}\left(\mathbb{R}^{2}\right)$ denote the homomorphism defining this action. Note that $G^{\prime \prime}$ is torsion-free, but we do not yet know that $\sigma$ is faithful, and therefore we don't know that $\sigma\left(G^{\prime \prime}\right)$ is torsion-free.

Let $K$ be the kernel of $\sigma$. Build a space $X$ from a $K\left(G^{\prime}, 1\right)$ and from $N$ by gluing along subsurfaces representing the subgroups $S_{m}$. Let $\widehat{X}$ be the cover 
of $X$ corresponding to $K$. Since $\sigma$ is injective on $G^{\prime}, \widehat{X}$ is obtained from copies of the cover $\widehat{N}$ of $N$ where $\pi_{1}(\widehat{N})=K \cap \pi_{1}(N)$, glued along disks in copies of the universal cover of $K\left(G^{\prime}, 1\right)$. But the universal cover of a $K(\pi, 1)$ is contractible, so $\widehat{X}$ is homotopic to a 3 -manifold, obtained from copies of $\widehat{N}$ of $N$ by attaching 1 -handles. In particular, $K$ is the fundamental group of an irreducible $3-$ manifold. It follows that every finitely-generated subgroup of $K$ is the fundamental group of a compact irreducible 3-manifold with boundary, by the Scott core theorem (see [15]). As [1] observed, every compact irreducible 3manifold with boundary has locally indicable fundamental group, and therefore by Theorem 2.1.11, has LO fundamental group. Since $K$ is locally LO, it is LO, by Lemma 2.1.6. We now show how to modify $\sigma$ to make it faithful.

Pick a point $p$, and blow up its orbit. That is, replace each translate $g(p)$ of $p$ by a closed disk $D_{g}$ in such a way that the diameters of these disks in any compact region go to zero. Since $\sigma$ is $C^{1}$ at $p$, we can extend the action of $G^{\prime \prime}$ on $\mathbb{R}^{2} \backslash G^{\prime \prime}(p)$ to the boundary circle $\partial D_{g}$ by the projective linear action on the unit tangent bundle $U T_{g(p)} \mathbb{R}^{2}$. Let $G_{p}^{\prime \prime}$ be the stabilizer of $p$ in $G^{\prime \prime}$. Then $\sigma\left(G_{p}^{\prime \prime}\right)$ is circularly-orderable, and the stabilizer of $\partial D_{g}$ in $\sigma\left(G_{p}^{\prime \prime}\right)$ is leftorderable, by Lemma 3.2.2. Let $K_{p}$ be the stabilizer of $\partial D_{g}$ in $G_{p}^{\prime \prime}$, so that $\sigma\left(K_{p}\right)$ is the stabilizer of $\partial D_{g}$ in $\sigma\left(G_{p}^{\prime \prime}\right)$. Since $K$ is left-orderable, and $\sigma\left(K_{p}\right)$ is left-orderable, it follows that $K_{p}$ is left-orderable by Lemma 2.1.9, and $G_{p}^{\prime \prime}$ is circularly-orderable, by Lemma 2.2.12. We can insert a faithful action of $G_{p}^{\prime \prime}$ on $D_{g}$ by thinking of $D_{g}$ as the cone on $S^{1}$, and coning the (positive) projectivized linear action of $G_{p}^{\prime \prime}$ on $S^{1}$ by a faithful action of $K_{p}$ on $I$, which exists by Theorem 2.2.14. We can then translate this action to the other disks $D_{h}$. The resulting action defines a faithful representation $\sigma^{\prime}$. Notice that even though $\sigma^{\prime}$ is only $C^{0}$, we could not perform the blow-up without knowing that $\sigma$ was $C^{1}$ at $p$. So $\sigma^{\prime}$ exhibits $G^{\prime \prime}$ as a torsion-free subgroup of $\mathrm{Homeo}^{+}\left(\mathbb{R}^{2}\right)$.

If there were a representation $\rho^{\prime \prime}: G^{\prime \prime} \rightarrow \operatorname{Homeo}^{+}\left(S^{1}\right)$ extending $\rho^{\prime}$, then the circular Euler class of $\rho_{-4 m}\left(F_{2}\right)$ would be equal to the circular Euler class of $S_{m}$, which as we calculated before, is $\leq-4 m+4 \log m a$, violating the MilnorWood inequality (Theorem 2.4.2). This contradiction shows that $\rho^{\prime \prime}$ cannot exist, and $G^{\prime \prime}$ is not circularly-orderable, as claimed.

Remark 4.4.4 Notice that in order to find a $C^{\infty}$ example, one just needs to find a torsion-free $C^{\infty}$ homology from $S_{m}$ to $\rho_{-4 m}\left(F_{2}\right)$. Such a homology can actually be constructed by hand, but the construction is somewhat complicated and unenlightening, so we do not include it here. 


\subsection{Homological rigidity for $\mathbb{Z} \oplus \mathbb{Z}$ actions}

In the remainder of the paper, we will show, in contrast to theorem $\mathrm{C}$, and Example 4.2.1 that for $C^{1}$ actions of $\mathbb{Z} \oplus \mathbb{Z}$, the Euler class always vanishes.

We do this by studying the possible dynamics of two commuting $C^{1}$ homeomorphisms $\alpha, \beta$, examining the cases based on the dynamics of $\beta$ on the fix point set of $\alpha$.

First we treat the case that $\alpha$ has a fixed point whose orbit under $\beta$ is not proper.

Lemma 4.5.1 Let $\rho: \mathbb{Z} \oplus \mathbb{Z} \rightarrow$ Homeo $^{+}\left(\mathbb{R}^{2}\right)$ be $C^{1}$, and suppose that $p$ has nontrivial stabilizer. If $p$ is fixed by a finite index subgroup, or has an indiscrete orbit, then $\rho^{*}([e])$ is trivial.

Proof Suppose the stabilizer of $p$ is a finite index subgroup. Since any finite index subgroup of $\mathbb{Z} \oplus \mathbb{Z}$ is isomorphic to $\mathbb{Z} \oplus \mathbb{Z}$, and since the Euler class is multiplicative under covers, it suffices to assume that $p$ is a global fixed point. In this case the Euler class of the planar action is equal to the Euler class of the (projective) action on the unit tangent bundle at $p$ obtained by linearizing. To see this, use Construction 4.1.2 for a constant developing map. In particular, the Euler class is pulled back from an action on $S^{1}$, and therefore by the Milnor-Wood inequality, the Euler class vanishes.

If $p$ is fixed by some element $\alpha$, then after passing to a finite index subgroup, we can assume $\alpha$ is a generator. Fix some other generator $\beta$ and let $p_{i}=\beta^{i}(p)$. Suppose $\left\{p_{i}\right\}$ is indiscrete and contains some subsequence which accumulates at $q$, which is fixed by $\alpha$. Some subsequence approaches $q$ radially along some tangent vector $v$. Since the action is $C^{1}$, it follows that $\alpha$ fixes $q$ and $d \alpha$ fixes $v$.

Let $p_{i}, p_{j}$ be very close to $q$, and let $\tau$ be an arc joining $p_{i}$ to $p_{j}$ so that $\tau^{\prime}(t)$ is nearly parallel to $v$ for all $t \in \tau$, and $d \beta^{j-i} \tau^{\prime}(0)=\tau^{\prime}(1)$. Then $d \alpha \tau^{\prime}(0)$ is very close to $\tau^{\prime}(0)$, and since $\alpha$ and $\beta$ commute, $d \alpha \tau^{\prime}(1)$ is very close to $\tau^{\prime}(1)$. Moreover, near $q, \alpha$ is very close in the $C^{1}$ topology to a linear transformation. So, since $d \alpha$ almost fixes $\tau^{\prime}(0)$ and $\tau^{\prime}(1)$, either these vectors are almost parallel, in which case we can take $\tau$ very close to a radial straight arc, or else $d \alpha$ is very close to the identity. In either case, for our choice of $\tau$, the arc $\alpha(\tau)$ is very close in the $C^{1}$ topology to $\tau$. We pass to the finite index subgroup generated by $\alpha$ and $\beta^{i-j}$. Again, since the Euler class is multiplicative under covers, it suffices to show that it vanishes in this case. We let $\tau \cup \alpha(\tau)$ be the 
image of $\partial P_{1}$ (where $P_{1}$ is a fundamental domain for our new copy of $\mathbb{Z} \oplus \mathbb{Z}$ ) under a "degenerate" developing map $d_{\rho}$ as in Construction 4.1.2. Then by our geometric reasoning, the index is 1 , and the Euler class, evaluated on the fundamental class of $\mathbb{Z} \oplus \mathbb{Z}$, is $1+1-2=0$, as claimed.

\subsection{The Alexander series of $\rho$}

In this section we prove that if $\alpha$ fixes some point $p$, then if there exists $\tau$ from $p$ to $\beta(p)$ which has algebraic intersection number zero with its translates $\beta^{i}(\tau)$, then the Euler class is zero. An individual treatment of this case is not logically necessary for the proof of theorem $\mathrm{D}$, but it does make subsequent sections easier to understand. Throughout this section and the next, we assume that the orbit of $p$ under $\langle\beta\rangle$ is proper (that is, it has no accumulation points) since otherwise, we could apply Lemma 4.5.1.

We fix the following notation. Let $p_{i}=\beta^{i}(p)$, and let $\tau$ be a smooth arc from $p_{0}$ to $p_{1}$ such that $d \beta\left(\tau^{\prime}(0)\right)=\tau^{\prime}(1)$. Let $P=\cup_{i} p_{i}$ and $l=\cup_{i} \beta^{i}(\tau)$. We also introduce notation $\delta=\alpha(\tau)$.

First we introduce an algebraic tool to study the dynamics of $\alpha$ and $\beta$, which we call the Alexander series, by analogy with the usual Alexander polynomial of a knot. The condition we will impose on $\tau$ will ensure that the series is actually a Laurent polynomial.

We assume the Euler class of our representation $\rho$ is $n \neq 0$. Look at the closed curve $\tau \cup \delta$. This is the image of a fundamental domain for the torus under a "degenerate" developing map, so we can calculate the Euler class of $\rho$ from the formula in Construction 4.1.2.

The curves $\tau, \delta$ both represent elements in relative homology

$$
[\tau],[\delta] \in H_{1}\left(\mathbb{R}^{2}, P ; \mathbb{Z}\right) .
$$

Since there is no natural intersection form on this group, we must work harder to find the correct algebraic definition of the Euler class.

There is a natural Laurent series associated to the action $\rho$, defined as follows:

Definition 4.6.1 The Alexander series of $\rho$, denoted

$$
A_{\rho} \in \mathbb{Z}\left[\left[t, t^{-1}\right]\right]
$$

is defined as follows. For the moment, assume that for our choice of $\tau$, that

$\delta$ and $\tau$ are transverse at 0 . The constant term is the algebraic intersection 
number, for a generic choice of representative (subject to the constraints above) of $\delta$ and $\tau$, plus the algebraic sign of the intersection at 0 . We now define the other coefficients. For $i \neq 0$, let the coefficient of $t^{i}$ be the algebraic intersection number of the interior of $\delta$ with the interior of $\beta^{i}(\tau)$.

If $d \alpha(p)=\mathrm{Id}$, so that $\tau$ and $\delta$ cannot be made transverse at 0 , we let $\phi$ be a diffeomorphism centered at $p$ with small support, which fixes $p$ and has linear part $d \phi(p)$ a nontrivial rotation. Then define

$$
\delta^{\prime}=\beta \phi \beta^{-1} \phi(\delta)
$$

and compute the constant term as above for $\delta^{\prime}$ and $\tau$. We denote the $i$ th coefficient of $A_{\rho}$ by $a_{i}$.

Notation 4.6.2 If $\mu, \nu$ are two arcs or loops in general position, we denote their algebraic intersection number by $\mu \cdot \nu$. So, with this notation, we have

$$
a_{i}=\delta \cdot \beta^{i}(\tau)
$$

and

$$
\mu \cdot \nu=-\nu \cdot \mu
$$

Remark 4.6.3 As defined, $A_{\rho}$ might depend on $\tau$. Nevertheless we suppress this in the notation.

Notice that the coefficients of the power series only depend on the germs of $\tau, \delta$ at $p_{0}, p_{1}$, together with their smooth isotopy class in $\mathbb{R}^{2} \backslash N\left(p_{0} \cup p_{1}\right)$, where $N\left(p_{0} \cup p_{1}\right)$ denotes a sufficiently small regular neighborhood of $p_{0} \cup p_{1}$. So if we replace $\tau, \delta$ with $\tau^{\prime}, \delta^{\prime}$ which are smoothly isotopic by an isotopy supported outside a neighborhood of $P$, the algebraic intersection number of $\delta^{\prime}$ with $\beta^{i}\left(\tau^{\prime}\right)$ is equal to the $i$ th coefficient of $A_{\rho}$.

For the moment, we have defined $A_{\rho} \in \mathbb{Z}\left[\left[t, t^{-1}\right]\right]$. We show, under the right conditions, that $A_{\rho}$ is actually a Laurent polynomial.

But first we must see how to calculate the Euler class of $\rho$ from the configuration of $\tau$ and $\delta$.

Lemma 4.6.4 Let $\rho: \mathbb{Z} \oplus \mathbb{Z} \rightarrow$ Homeo $^{+}\left(\mathbb{R}^{2}\right)$ be $C^{1}$. Let $\alpha, \beta$ be the generators, and let $p$ be fixed by $\alpha$. Let $\tau$ be an embedded arc from $p$ to $\beta(p)$, and let $\delta$ be $\alpha(\tau)$. Let $\tau^{ \pm}$be immersed proper rays in $\mathbb{R}^{2}$ such that $\tau^{-} \cup \tau \cup \tau^{+}$ is an immersed proper line, and $\tau^{ \pm}$intersect $\tau$ only at their endpoints. Then the Euler class $\rho^{*}([e])$, evaluated on the fundamental cycle of $\mathbb{Z} \oplus \mathbb{Z}$, satisfies the formula

$$
\rho^{*}([e])([\mathbb{Z} \oplus \mathbb{Z}])=\delta \cdot \tau^{+}-\delta \cdot \tau^{-} .
$$


Proof First we prove the lemma in the case that $\tau^{+}, \tau^{-}$are properly embedded, and intersect $\tau$ only at their endpoints.

For concreteness, let $\tau^{-} \cup \tau \cup \tau^{+}$be the real axis, oriented so that $\tau^{-}$limits to $-\infty$.
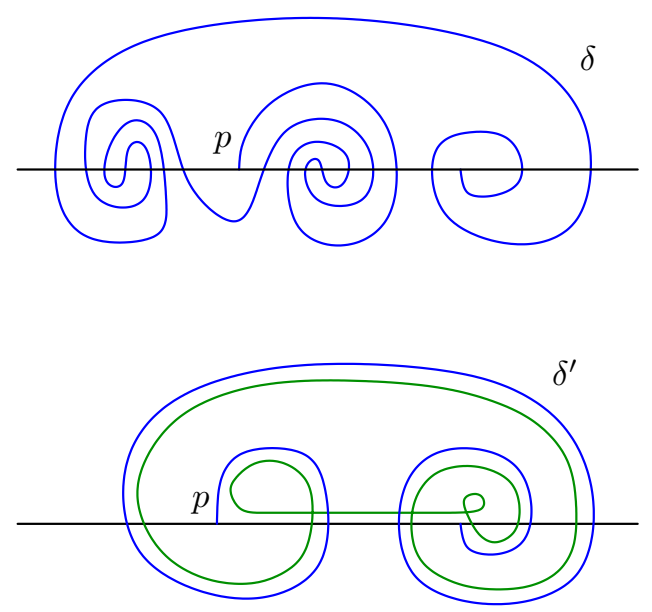

Figure 4: The Euler class can be calculated from the intersection numbers $\delta \cdot \tau^{ \pm}$. The green loop is the smoothed boundary of a fundamental domain for the hypothetical $\mathbb{Z} \oplus \mathbb{Z}$ action. The index of the green loop is $\delta \cdot \tau^{+}-\delta \cdot \tau^{-}+1=2+1+1=4$, and the Euler class is 3 .

We can deform $\delta$ through a family of smooth arcs which are all embedded, which do not intersect $p$ or $\beta(p)$ except at their endpoints, and whose germ near $p, \beta(p)$ is fixed. The end result of this deformation we will denote $\delta^{\prime}$. This deformation does not change either the index of $\delta \cup \tau$, or the intersection numbers $\delta \cdot \tau^{ \pm}$. After such a deformation we can assume $\delta^{\prime}$ is of a particularly simple form: it consists of three pieces: a concentric spiral around $p$ through $\delta \cdot \tau^{-}$revolutions, a concentric spiral around $\beta(p)$ through $\delta \cdot \tau^{+}$revolutions, and an arc between the two which has no vertical tangent. To see this, observe that we can deform $\delta$ to $\tau$ by an isotopy rel. endpoints through smooth arcs, at the cost of twisting the tangent vectors at $p$ and at $\beta(p)$ through $\delta \cdot \tau^{-}$and $\delta \cdot \tau^{+}$revolutions, respectively. Then we can "undo" this twisting by inserting spirals, to produce $\delta^{\prime}$.

When we smooth the curve $\tau \cup \delta$ to calculate the index by the formula of Construction 4.1.2, we introduce another twist at $\beta(p)$. As described in Construction 4.1.2, this is because to smooth the curve correctly, we must make a perturbation at the degenerate edges of the developing map, in a way which 
is coherent with respect to the edge pairing transformations. If the "degenerate edge" from $p$ to $\alpha(p)$ is perturbed to a short vertical edge lying on the positive side of $\tau$, then the degenerate edge from $\beta(p)$ to $\alpha \beta(p)$ must also be on the positive side of $\tau$, and the end of $\delta^{\prime}$ must cross over $\tau$ near $\beta(p)$ to reach $\alpha \beta(p)$. It follows that the index of the smoothing of $\tau \cup \delta$ is equal to $\delta \cdot \tau^{+}-\delta \cdot \tau^{-}+1$ and therefore, by the formula of Construction 4.1.2, the Euler class is $\delta \cdot \tau^{+}-\delta \cdot \tau^{-}$, as claimed.

For an example, see Figure 4. In this case $\delta \cdot \tau^{+}=2, \delta \cdot \tau^{-}=-1$ and the Euler class is 3 .

Note that this calculation is still valid if $\tau^{-}, \tau^{+}$are merely properly immersed rays which do not intersect $\tau$ except at their endpoints. For, we can always isotope $\delta$ to $\delta^{\prime}$ which is contained in a neighborhood of $\tau$, without affecting the intersection number with $\tau^{+}$or $\tau^{-}$, and therefore the calculation is insensitive to intersections of $\tau^{ \pm}$with each other or with themselves away from $\tau$. This completes the proof in general.

With this lemma, we can establish the following fundamental properties of $A_{\rho}$.

Lemma 4.6.5 Suppose that $\tau$ has the property that for any $i$, the algebraic intersection number of $\tau$ and $\beta^{i}(\tau)$ is zero. Then the Alexander series $A_{\rho}(t)=$ $\sum_{i} a_{i} t^{i}$ has the following properties:

(1) $A_{\rho}$ is actually a Laurent polynomial. That is, the coefficient of $t^{i}$ is zero for all but finitely many $i$.

(2) The sum of the coefficients is zero:

$$
A_{\rho}(1)=0 .
$$

(3) The Euler class $\rho^{*}([e])([\mathbb{Z} \oplus \mathbb{Z}])$, which we abbreviate by $e_{\rho}$, satisfies

$$
e_{\rho}=\sum_{i>0} a_{i}-\sum_{i<0} a_{i} .
$$

In this case, we call $A_{\rho}$ the Alexander polynomial of $\rho$.

Proof Recall our standing assumption throughout this section that the orbit of $p$ under $\langle\beta\rangle$ is discrete, since otherwise Lemma 4.5.1 would apply. We show how the first fact of the lemma follows from this assumption. By the assumption, for large $i$, the points $p_{i}, p_{i+1}$ escape any compact set. Since $\beta^{i}(\tau)$ is an arc from $p_{i}$ to $p_{i+1}$, the algebraic intersection number of this arc with $\tau \cup \delta$ is zero. But by hypothesis, $\beta^{i}(\tau)$ and $\tau$ have algebraic intersection number zero, so the algebraic intersection with $\delta$ alone is also zero. 
The second fact follows for essentially the same reason as the first fact: the union $\tau \cup \delta$ is an (immersed) closed loop, and the sum of coefficients is equal to the algebraic intersection number of $\tau \cup \delta$ with the union $l=\cup_{i} \beta^{i}(\tau)$. Since the $p_{i}$ are unbounded, the algebraic intersection of the loop and $l$ is zero, and therefore the formula follows.

The third fact follows from Lemma 4.6.4, by using $\bigcup_{i<0} \beta^{i}(\tau)$ as $\tau^{-}$, and $\bigcup_{i>0} \beta^{i}(\tau)$ as $\tau^{+}$. Now, the union $\bigcup_{i} \beta^{i}(\tau)$ is not necessarily properly immersed in general, but note that we can homotope each $\beta^{i}(\tau)$ rel. a neighborhood of its endpoints (not necessarily equivariantly!) without intersecting the endpoints of $\tau$ during the track of the homotopy, to new $\tau_{i}^{\prime}$ until the union $\bigcup_{i} \tau_{i}^{\prime}$ is properly immersed. This does not change the algebraic intersection number with $\delta$. Then

$$
e_{\rho}=\sum_{i>0} \delta \cdot \tau_{i}^{\prime}-\sum_{i<0} \delta \cdot \tau_{i}^{\prime}=\sum_{i>0} \delta \cdot \beta^{i}(\tau)-\sum_{i<0} \delta \cdot \beta^{i}(\tau)=\sum_{i>0} a_{i}-\sum_{i<0} a_{i} .
$$

With this algebraic tool, we can now prove the following lemma:

Lemma 4.6.6 Let $\rho: \mathbb{Z} \oplus \mathbb{Z} \rightarrow$ Homeo $^{+}\left(\mathbb{R}^{2}\right)$ be a $C^{1}$ action which is not free. Suppose $\mathbb{Z} \oplus \mathbb{Z}$ is generated by $\alpha$ and $\beta$, where $\alpha$ fixes some point $p$, and there is a smooth embedded arc $\tau$ from $p$ to $\beta(p)$ with $d \beta\left(\tau^{\prime}(0)\right)=\tau^{\prime}(1)$ such that the algebraic intersection number of $\tau$ with $\beta^{i}(\tau)$ is zero for any $i$. Then the Euler class $\rho^{*}([e])$ is zero.

Proof Let $a_{i}$ be the $i$ th coefficient of $A_{\rho}$. Let $G_{n}$ be the subgroup of $\mathbb{Z} \oplus \mathbb{Z}$ of index $2 n+1$ generated by $\alpha$ and $\beta^{2 n+1}$. This gives a new representation $\rho_{n}$ of $\mathbb{Z} \oplus \mathbb{Z}$, whose Euler class is equal to $2 n+1$ times the Euler class of $\rho$, by multiplicativity. The new edges $\tau_{2 n+1}, \delta_{2 n+1}$ which are the nondegenerate sides of a fundamental domain for the new $\mathbb{Z} \oplus \mathbb{Z}$ subgroup can be chosen to be the unions

$$
\tau_{2 n+1}=\bigcup_{i=-n}^{n} \beta^{i}(\tau), \quad \delta_{2 n+1}=\bigcup_{i=-n}^{n} \beta^{i}(\delta)
$$

respectively.

By the definition of $A_{\rho}$ and the fact that

$$
\beta^{i}(\delta) \cdot \beta^{j}(\tau)=\delta \cdot \beta^{j-i}(\tau)=a_{j-i}
$$

we can calculate

$$
A_{\rho_{n}}(t)=\sum_{j} \sum_{i=-2 n}^{2 n}(2 n+1-|i|) a_{i+j(2 n+1)} t^{j}
$$


and so by Lemma 4.6.5, we have

$$
\begin{aligned}
e_{\rho_{n}} & =(2 n+1) e_{\rho} \\
= & \sum_{j>0} \sum_{i=-2 n}^{2 n}(2 n+1-|i|) a_{i+j(2 n+1)}-\sum_{j<0} \sum_{i=-2 n}^{2 n}(2 n+1-|i|) a_{i+j(2 n+1)} \\
& =\sum_{i>0} \min (i, 2 n+1) a_{i}-\sum_{i<0} \min (-i, 2 n+1) a_{i} .
\end{aligned}
$$

Now, by Lemma 4.6.5, the coefficients $a_{i}$ are zero for $|i|$ sufficiently large. But one sees that the coefficient of each $a_{i}$ in the expression for $e_{\rho_{n}}$ is eventually constant and equal to $i$, so as $n$ increases, the right hand side is eventually constant. On the other hand, it is equal to $(2 n+1) e_{\rho}$, so $e_{\rho}$ is equal to zero, as claimed

\subsection{The case that $\tau \cdot \beta^{i}(\tau) \neq 0$}

In general, we cannot find an arc $\tau$ with the properties of Lemma 4.6.6. An example is constructed in [14] of an orientation-preserving homeomorphism $h$ of $\mathbb{R}^{2}$ with a proper orbit $h^{i}(p)$ such that every arc $\tau$ from $p$ to $h(p)$ must intersect its translates. We show how to modify our arguments to deal with the case that $\tau \cdot \beta^{i}(\tau) \neq 0$ for some (possibly infinitely many) $i$.

Let $\tau$ be any smooth arc joining $p$ to $\beta(p)$, satisfying $d \beta\left(\tau^{\prime}(0)\right)=\tau^{\prime}(1)$.

Definition 4.7.1 The self-intersection series, denoted $B_{\rho} \in \mathbb{Z}\left[\left[t, t^{-1}\right]\right]$ is defined by setting the constant term equal to zero, and for $i \neq 0$, the $t^{i}$ term equal to the algebraic intersection number of $\tau$ with $\beta^{i}(\tau)$.

There is no good way to define the homological self-intersection of $\tau$ with itself, so the constant term must be zero. A meaningful quantity to describe the smooth isotopy class of $\tau$ rel. endpoints is the writhe, defined as follows:

Definition 4.7.2 Let $\tau$ be a smooth immersed arc in $\mathbb{R}^{2}$. Choose an orientation-preserving identification of tangent spaces

$$
\phi: T_{\tau(0)} \mathbb{R}^{2} \longrightarrow T_{\tau(1)} \mathbb{R}^{2}
$$

such that $\phi\left(\tau^{\prime}(0)\right)=\tau^{\prime}(1)$. Let $\mathscr{C}$ denote the space of homotopy classes of $C^{1}$ immersed curves $\sigma$ in $\mathbb{R}^{2}$ from $\tau(0)$ to $\tau(1)$ with $\phi\left(\sigma^{\prime}(0)\right)=\sigma^{\prime}(1)$. Note that these curves may intersect their endpoints in their interiors. The class of $\tau$ in $\mathscr{C}$ is denoted $[\tau]$ and is called the writhe of $\tau$. 
Given a group $G$, an affine space for $G$ is a space $X$ with a transitive $G$ action whose point stabilizers are trivial. For example, the action of a group $G$ on itself makes $G$ into an affine space for $G$. Similarly, the fibers of a principle $G$-bundle are affine spaces for $G$.

Given two smooth 1-manifolds $\kappa_{1}, \kappa_{2}$ in the plane, the connect sum is obtained as follows. Let $I$ be an embedded arc joining a point in the interior of $\kappa_{1}$ to a point in the interior of $\kappa_{2}$. Thicken $I$ to a 1 -handle $I \times I$, with boundary $I \times\{0,1\} \cup\{0,1\} \times I$, where $I \times\{0,1\}$ is contained in the $\kappa_{i}$. Then the connect sum $\kappa_{1} \sharp \kappa_{2}$ is given by the formula

$$
\kappa_{1} \sharp \kappa_{2}=\left(\kappa_{1} \cup \kappa_{2}\right) \cup \partial(I \times I) \backslash\left(\kappa_{1} \cup \kappa_{2}\right) \cap \partial(I \times I) .
$$

This produces a 1-manifold with corners; we round the corners to get a smooth 1 -manifold which by abuse of notation we call the connect sum. See Figure 5 for an illustration of this operation.

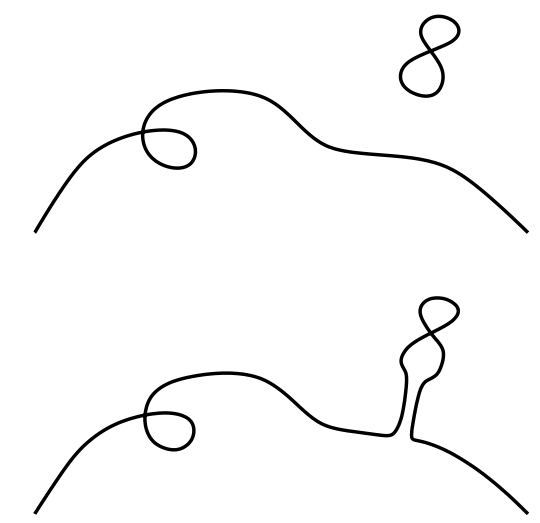

Figure 5: The operation of connect summing with a small figure 8 on the positive side generates the action of $\mathbb{Z}$ on $\mathscr{C}$.

Lemma 4.7.3 The space $\mathscr{C}$ is an affine space for $\mathbb{Z}$ where the action of 1 is given by connect summing with a small figure 8 on the positive side.

Proof This is a very special case of Smale's immersion theorem [35]. In this context, it basically follows from the Whitney trick [41]. The Gauss map takes a $C^{1}$ immersed arc to the unit circle in $\mathbb{R}^{2}$. As a family of arcs varies in an equivalence class of $\mathscr{C}$, the images vary in a homotopy class rel. endpoints. The space of homotopy classes of maps from $I$ to $S^{1}$ with fixed endpoints is an affine space for $\mathbb{Z}$; the content of Smale's theorem in this case is that these two spaces are equivalent. 
Note that there is no natural basepoint for $\mathscr{C}$, but given $\tau$ and $\delta$, the difference of the writhe of $[\tau]$ and $[\delta]$ is an integer, which we denote by

$$
[\tau]-[\delta]=n \in \mathbb{Z}
$$

where we are using the structure of $\mathscr{C}$ as an affine $\mathbb{Z}$ space. That is, if $n$ is non-negative (say), then the connect sum of $\tau$ with $n$ copies of a small figure 8 on the positive side is in the same equivalence class of $\mathscr{C}$ as $\delta$.

Lemma 4.7.4 With $\tau, \delta$ as above, the difference in writhe is equal to the Euler class:

$$
[\tau]-[\delta]=e_{\rho}
$$

Proof This follows immediately from the definition of index and the formula in Construction 4.1.2. Firstly, observe that the index of the smoothing only depends on the class of $\tau$ and $\delta$ in $\mathscr{C}$. If $\tau=\delta$, then the index is obviously 1 , and $e_{\rho}=0$. Finally, connect summing $\tau$ with a figure 8 on the positive side changes both the writhe of $[\tau]$ and the index of the smoothing by 1 .

For $\tau$ with the properties of the previous section, $B_{\rho}$ is identically zero. The Alexander series $A_{\rho}$ is defined as before. We now prove the analogous case of Lemma 4.6.5 where $B_{\rho}$ is not identically zero. We prove the following lemma for arbitrary $\tau$ joining $p$ to $\beta(p)$; in particular, we do not assume $\tau$ is embedded.

Lemma 4.7.5 For general $\tau$, the series $A_{\rho}$ and $B_{\rho}$ satisfy the following properties:

(1) The coefficients of $B_{\rho}$ are antisymmetric; that is, if $B_{\rho}=\sum_{i} b_{i} t^{i}$, then

$$
b_{i}=-b_{-i} \text {. }
$$

(2) The sum $A_{\rho}-B_{\rho}$ is a Laurent polynomial.

(3) The sum of the coefficients of $A_{\rho}-B_{\rho}$ is zero:

$$
\left(A_{\rho}-B_{\rho}\right)(1)=0 .
$$

(4) The Euler class $\rho^{*}([e])([\mathbb{Z} \oplus \mathbb{Z}])$, which we abbreviate by $e_{\rho}$, satisfies

$$
e_{\rho}=\sum_{i>0}\left(a_{i}-b_{i}\right)-\sum_{i<0}\left(a_{i}-b_{i}\right)
$$


Proof The first fact follows because the intersection number of $\tau$ with $\beta^{i}(\tau)$ is equal to the intersection number of $\beta^{-i}(\tau)$ with $\tau$, and intersection number of 1 -cycles is antisymmetric.

The second and third facts follow as in Lemma 4.6.5, by the fact that $\tau \cup \delta$ (where $\tau$ has the opposite orientation) is an (immersed) closed loop, and the orbit $\beta^{i}(p)$ is proper.

To calculate the Euler number, we move $\tau$ and $\delta$ by a homotopy $\tau_{t}, \delta_{t}$ through $C^{1}$ immersions whose germs near $p$ and $\beta(p)$ are constant. We move $\tau, \delta$ equivariantly, so that at each stage, $\delta_{t}=\alpha\left(\tau_{t}\right)$. We also connect sum $\tau$ and $\delta$ equivariantly with sufficiently many figure 8 's on the positive or negative sides until they are embedded curves from $p$ to $\beta(p)$.

Note that at finitely many times $t_{0}$, we might move $\tau_{t_{0}}$ through some $p_{i}$, and change $b_{i}$ and $b_{i-1}$. However, since $\delta_{t_{0}}=\alpha\left(\tau_{t_{0}}\right)$, it follows that $\delta_{t_{0}}$ passes through $p_{i}$ simultaneously. Therefore $\tau_{t} \cdot \beta^{i}(\tau)$ and $\delta_{t} \cdot \beta^{i}(\tau)$ change by the same amount at such a time $t_{0}$, and therefore the differences $\delta_{t} \cdot \beta^{i}(\tau)-\tau_{t} \cdot \beta^{i}(\tau)$ are always constant, and equal to $a_{i}-b_{i}$.

We also connect sum $\tau$ and $\delta$ equivariantly with sufficiently many small figure 8 's on positive or negative sides. This does not affect any $a_{i}$ or $b_{i}$, and by Lemma 4.7.4, it does not affect the difference in writhe $\left[\tau_{t}\right]-\left[\delta_{t}\right]$ or the calculation of the Euler class.

It follows that the Euler class can be calculated from the formula given in Lemma 4.6.4, after finding suitable properly immersed rays $\tau^{ \pm}$such that $\tau^{-} \cup$ $\tau \cup \tau^{+}$is a properly immersed line, and $\tau^{ \pm}$do not intersect $\tau$ except at their endpoints.

We obtain such rays $\tau^{ \pm}$by a suitable deformation of the rays $\bigcup_{i<0} \beta^{i}(\tau)$ and $\bigcup_{i>0} \beta^{i}(\tau)$. Initially, some $\beta^{i}(\tau)$ might intersect $\tau$ essentially. But we can homotope each $\beta^{i}(\tau)$ through smooth curves, rel. endpoints, to $\tau_{i}^{\prime}$ which are disjoint from $\tau$, at the cost of crossing $\tau_{i}^{\prime} b_{i}$ times (algebraically) over $p, \beta(p)$. Then for each $i$,

$$
\delta \cdot \tau_{i}^{\prime}-\tau \cdot \tau_{i}^{\prime}=\delta \cdot \beta^{i}(\tau)-\tau \cdot \beta^{i}(\tau) .
$$

Moreover, we can homotope $\tau_{i}^{\prime}$ through immersions rel. endpoints, and without crossing $p$ or $\beta(p)$, to some new $\tau_{i}^{\prime \prime}$, so that the unions $\bigcup_{i>0} \tau_{i}^{\prime \prime}=\tau^{+}$and $\bigcup_{i<0} \tau_{i}^{\prime \prime}=\tau^{-}$are properly immersed, and do not intersect $\tau$ except at their endpoints. Then

$$
\delta \cdot \tau_{i}^{\prime \prime}=\delta \cdot \beta^{i}(\tau)-\tau \cdot \beta^{i}(\tau)=a_{i}-b_{i}
$$

so the formula for the Euler class follows.

Note that since $A_{\rho}-B_{\rho}$ is a Laurent polynomial, this is actually a finite sum. 
Having established the properties of $A_{\rho}$ and $B_{\rho}$, we can now prove the analogue of Lemma 4.6.6 for arbitrary $\tau$.

Lemma 4.7.6 Let $\rho: \mathbb{Z} \oplus \mathbb{Z} \rightarrow$Homeo $^{+}\left(\mathbb{R}^{2}\right)$ be a $C^{1}$ action which is not free. Then the Euler class $\rho^{*}([e])$ is zero.

Proof Without loss of generality, we can assume $\alpha$ fixes some $p$, and the orbit of $p$ under $\beta$ is proper. Let $\tau$ be as above, and define the series $A_{\rho}, B_{\rho}$. Let $G_{n}$ be the subgroup of $\mathbb{Z} \oplus \mathbb{Z}$ of index $2 n+1$ generated by $\alpha$ and $\beta^{2 n+1}$ as before. As in the proof of Lemma 4.6.6, we have

$$
\begin{aligned}
e_{\rho_{n}}=(2 n+1) e_{\rho} & =\sum_{j>0} \sum_{i=-2 n}^{2 n}(2 n+1-|i|)\left(a_{i+j(2 n+1)}-b_{i+j(2 n+1)}\right) \\
& -\sum_{j<0} \sum_{i=-2 n}^{2 n}(2 n+1-|i|)\left(a_{i+j(2 n+1)}-b_{i+j(2 n+1)}\right) .
\end{aligned}
$$

On the other hand, by Lemma 4.7.5, the coefficients $a_{i}-b_{i}$ are zero for $|i|$ sufficiently large, so as $n$ increases, the right hand side is eventually constant. On the other hand, this sum is equal to $(2 n+1) e_{\rho}$, so $e_{\rho_{n}}=e_{\rho}=0$, as claimed.

\subsection{Vanishing of the Euler class}

From the previous section, we know that a $C^{1} \mathbb{Z} \oplus \mathbb{Z}$ action with nontrivial Euler class must be free; that is, no nontrivial element has a fixed point.

We make use of several theorems, one of which was originally proved by Brouwer (see [2]), but later given a simpler and more illuminating proof by many people including [33], [10]. The first theorem says that for $\beta$ a fixed-point-free orientation preserving homeomorphism of $\mathbb{R}^{2}$, and $p$ arbitrary, we can find an arc $\tau$ from $p$ to $\beta(p)$ which does not intersect its translates, except at the endpoints. More formally,

Theorem 4.8.1 (Brouwer) Let $\alpha: \mathbb{R}^{2} \rightarrow \mathbb{R}^{2}$ be orientation-preserving and fixed point free. Then every point has a proper orbit, and for every point $p \in \mathbb{R}^{2}$ there is an embedded line $l \subset \mathbb{R}^{2}$ containing $p$, on which the action of $\alpha$ is conjugate to a translation.

Remark 4.8.2 The caveat of Theorem 4.8.1 is that the invariant line $l$ it produces through every point $p$ is not generally properly embedded, even though the orbit of every point is properly embedded. 
We also make use of a property of fixed-point-free orientation-preserving homeomorphism, established by Brown, in [3].

Brown makes the following definition:

Definition 4.8.3 An orientation-preserving homeomorphism $h: \mathbb{R}^{2} \rightarrow \mathbb{R}^{2}$ is free if for every bounded, connected set $X$ with $X \cap h(X)=\emptyset$, we have $X \cap$ $h^{i}(X)=\emptyset$ for all $i \in \mathbb{Z}$.

Theorem 4.8.4 (Brown) Let $h: \mathbb{R}^{2} \rightarrow \mathbb{R}^{2}$ be a fixed-point-free orientation preserving homeomorphism. Then $h$ is free.

Finally, we prove an algebraic lemma:

Lemma 4.8.5 Let $h: \mathbb{R}^{2} \rightarrow \mathbb{R}^{2}$ be a $C^{1}$ orientation preserving homeomorphism. Then for any two arcs $\tau_{1}, \tau_{2}$ from $p$ to $h(p)$ such that the union of translates of $\tau_{i}$ is a $C^{1}$ embedded line for $i=1,2$, the writhe of $\tau_{1}$ and $\tau_{2}$ are equal.

Proof Note that for an arc $\tau$ with the property in question, the homeomorphism $h$ satisfies $d h\left(\tau^{\prime}(0)\right)=\tau^{\prime}(1)$ so the difference in writhe of $\tau_{1}$ and $\tau_{2}$ make sense.

We go from $\tau_{1}$ to $\tau_{2}$ by a sequence of deformations of two different kinds.

The first kind of deformation does not change the writhe. This is a deformation $\tau_{t}$ through smooth embedded curves which for each $t$ satisfy $d h\left(\tau_{t}^{\prime}(0)\right)=\tau_{t}^{\prime}(1)$. For certain values of $t, \tau_{t}$ might pass through some vertex $h^{i}(p)$. By the embeddedness assumption, $i \neq 0,1$. Moreover, we choose the track of the isotopy of $\tau_{t}$ to be in general position with respect to each $h^{i}(p)$, so that for instance, there are only finitely many values of $t$ for which $\tau_{t}$ passes through some $h^{i}(p)$.

For any index $i$, for small values of $t-1$ we have $\tau_{t} \cdot h^{i}\left(\tau_{t}\right)=0$. As $\tau_{t}$ passes over some vertex $h^{i}(p)$, the value of $\tau_{t} \cdot h^{i}\left(\tau_{t}\right)$ changes by $e$, and the value of $\tau_{t} \cdot h^{i+1}\left(\tau_{t}\right)$ changes by $-e$, where $e= \pm 1$ depending on orientations. Simultaneously, the values of $h^{-i}\left(\tau_{t}\right) \cdot \tau_{t}$ and $h^{-i-1}\left(\tau_{t}\right) \cdot \tau_{t}$ change by $e$ and $-e$. No other intersection numbers change for nearby values of $t$. It follows that the finite sum

$$
w_{t}=\sum_{i>0} \tau_{t} \cdot h^{i}\left(\tau_{t}\right)-\sum_{i<0} \tau_{t} \cdot h^{i}\left(\tau_{t}\right)
$$

is constant under deformations of the first kind. 
The second kind of deformation changes the writhe. This consists of modifying $\tau_{t}$ in a small neighborhood of $p$ to some new $\tau_{t^{\prime}}$ by introducing a positive or negative "twist", thereby changing the writhe by \pm 1 . Under such a deformation

$$
\tau_{t^{\prime}} \cdot h^{-1}\left(\tau_{t^{\prime}}\right)-\tau_{t} \cdot h^{-1}\left(\tau_{t}\right)=1
$$

and

$$
\tau_{t^{\prime}} \cdot h\left(\tau_{t^{\prime}}\right)-\tau_{t} \cdot h\left(\tau_{t}\right)=-1
$$

for a positive twist, and the values change oppositely for a negative twist. It follows that $w_{t}$ (as defined in the previous paragraph) changes by \pm 2 under deformations of the second kind.

By combining deformations of these two kinds, we see that

$$
w_{t}=2\left(\left[\tau_{t}\right]-\left[\tau_{1}\right]\right)
$$

where as in Lemma 4.7.4 the notation $\left[\tau_{t}\right]-\left[\tau_{1}\right]$ means the difference in writhe. It follows that $w_{t}$ is equal to 0 iff the writhe of $\tau_{t}$ is equal to the writhe of $\tau_{1}$. On the other hand, if we deform $\tau_{1}$ to $\tau_{2}$ by a sequence of deformations of the two kinds above, then $w_{2}=0$, since $\tau_{2}$ does not intersect $h^{i}\left(\tau_{2}\right)$ except at its endpoints. So $\left[\tau_{2}\right]-\left[\tau_{1}\right]=0$, and the lemma is proved.

Remark 4.8.6 In fact, the argument of Lemma 4.6.6 can be used to give another proof of Lemma 4.8.5. Conversely, Lemma 4.8.5 can be used to give a different proof of Lemma 4.6.6. But the argument of Lemma 4.8.5 does not easily generalize to prove Lemma 4.7.6, whereas the argument of Lemma 4.6.6 does.

We are now ready to prove the main theorem on homological rigidity of $C^{1}$ $\mathbb{Z} \oplus \mathbb{Z}$ actions:

Theorem D Let $\rho: \mathbb{Z} \oplus \mathbb{Z} \rightarrow$ Homeo $^{+}\left(\mathbb{R}^{2}\right)$ be a $C^{1}$ action. Then the Euler class $\rho^{*}([e]) \in H^{2}(\mathbb{Z} \oplus \mathbb{Z} ; \mathbb{Z})$ is zero.

Proof By Lemma 4.7.6, we have proved this theorem except in the case that $\rho(\mathbb{Z} \oplus \mathbb{Z})$ is free.

Let $p$ be arbitrary, and let $\tau$ be a smooth arc from $p$ to $\beta(p)$ so that $\bigcup_{i} \beta^{i}(\tau)=l$ is an embedded line. Such a $\tau$ can be found by Theorem 4.8.1. Let $\sigma$ be an arc from $p$ to $\alpha(p)$. For each point $p_{t} \in \sigma$, we let $\tau_{t}$ be a smooth arc from $p_{t}$ to $\beta\left(p_{t}\right)$ so that $\bigcup_{i} \beta^{i}\left(\tau_{t}\right)=l_{t}$ is an embedded line. We show that for any $t_{0}$, there is an $\epsilon$ so that for $\left|t-t_{0}\right|<\epsilon$, the family $\tau_{t}$ can be chosen to vary smoothly. 
To see this, observe that we can certainly choose a smoothly varying family $\tau_{t}$ near $\tau_{t_{0}}$ so that $\tau_{t}$ intersects $\beta\left(\tau_{t}\right)$ only at $\beta\left(p_{t}\right)$. But by Theorem 4.8.4, this implies that the union of the $\beta^{i}\left(\tau_{t}\right)$ is an embedded line, as required.

It follows that we can subdivide $[0,1]$ into finitely many intervals

$$
\left[0, q_{1}\right],\left[q_{1}, q_{2}\right], \ldots,\left[q_{n}, 1\right]
$$

and choose smooth families $\tau_{t}^{i}$ for $q_{i} \leq t \leq q_{i+1}$ with the property that $\tau_{0}^{0}=\tau$ and $\tau_{1}^{n}=\alpha(\tau)$. But $\tau_{q_{i+1}}^{i}$ and $\tau_{q_{i+1}}^{i+1}$ have equal writhe, by Lemma 4.8.5, so we can insert a 1 -parameter family of isotopies from $\tau_{q_{i+1}}^{i}$ to $\tau_{q_{i+1}}^{i+1}$ whose germ at $p_{t}, \beta\left(p_{t}\right)$ is fixed. After inserting these isotopies, we have constructed a one parameter family of curves $\tau_{t}$ from $\tau$ to $\alpha(\tau)$ with $d \beta\left(\tau_{t}^{\prime}(0)\right)=\tau_{t}^{\prime}(1)$ for all $t$. The vector field $\tau_{t}^{\prime}$ pulls back to give a trivialization over a fundamental domain $P_{1}$ for the torus. It follows from the formula of Construction 4.1.2 that the Euler class is zero, and the theorem is proved.

Remark 4.8.7 It might be possible to try to prove theorem $\mathrm{D}$ by a cancellation argument, by analogy with Lemma 4.6.6 and Lemma 4.7.6. The problem is that, though the orbit of any point $p$ under any cyclic subgroup of a free action $\rho(\mathbb{Z} \oplus \mathbb{Z})$ is proper, it is not true that the orbit under all of $\rho(\mathbb{Z} \oplus \mathbb{Z})$ is proper.

Remark 4.8.8 Notice that the use of Brown's theorem in the proof of theorem $\mathrm{D}$ is essential, in order to be able to apply compactness of the interval $\sigma$ and compare the relative writhe of $\tau$ and $\beta(\tau)$. Brown's theorem itself is a kind of compactness theorem, since it lets one establish properties of the entire orbit of a connected set $\tau$ from the properties of only two translates $\tau$ and $\beta(\tau)$.

\section{References}

[1] S Boyer, D Rolfsen, B Wiest, Left-orderability of 3-manifold groups, preprint

[2] L E J Brouwer, Remark on the plane translation theorem, Nederl. Akad. Wetensch. Proc. 21 (1919) 935-936

[3] M Brown, Homeomorphisms of two-dimensional manifolds, Houston J. Math. 11 (1985) 455-469

[4] R Burns, V Hale, A note on group rings of certain torsion-free groups, Canad. Math. Bull. 15 (1972) 441-445

[5] D Calegari, Dynamical forcing of circular groups, to appear in Trans. AMS, arXiv:math.GT/0312066 
[6] C Carathéodory, Über die Begrenzung einfach zusammenhängender Gebiete, Math. Ann. 73 (1913) 323-370

[7] P Dehornoy, Braids and self-distributivity, Progress in Mathematics 192, Birkhäuser Verlag, Basel (2000)

[8] P Dehornoy, I Dynnikov, D Rolfsen, B Wiest, Why are braids orderable? Panoramas et Synthèses 14, Soc. Math. de France, Paris (2002)

[9] A Denjoy, Sur les courbes définies par les équations différentielles à la surface du tore, J. Math. Pures Appl. 11 (1932) 333-375

[10] J Franks, A new proof of the Brouwer plane translation theorem, Ergodic Theory Dynam. Systems 12 (1992) 217-226

[11] E Ghys, Groupes d'homéomorphismes du cercle et cohomologie bornée, in "The Lefschetz centennial conference, Part III (Mexico City, 1984)", Contemp. Math. 58, Amer. Math. Soc., Providence, RI (1987) 81-106

[12] E Ghys, Groups acting on the circle, Enseign. Math. (2) 47 (2001) 329-407

[13] E Ghys, private communication

[14] M Handel, A fixed point theorem for planar homeomorphisms, Topology 38 (1999) 235-264

[15] J Hempel, 3-manifolds, Ann. of Math. Studies 86, Princeton University Press (1976)

[16] D Husemoller, Fibre bundles, Third Edition, Grad. Texts in Math. 20 Springer-Verlag, Berlin (1994)

[17] S Jekel, A simplicial formula and bound for the Euler class, Israel Journal of Mathematics 66 (1989) 247-259

[18] H Kneser, Die Deformationssätze der einfach zusammenhängenden Flacher, Math. Zeit. 25 (1926) 362-372

[19] S Levy (editor), The Eightfold way: the beauty of Klein's quartic curve, MSRI publications 35, Cambridge University Press (1999)

[20] J-L Loday, Cyclic homology, Die Grundlehren der Mathematischen Wissenschaften 301, Springer-Verlag, Berlin (1998)

[21] S Mac Lane, Homology, Die Grundlehren der Mathematischen Wissenschaften 114, Springer-Verlag, Berlin (1963)

[22] J Mather, Topological proofs of some purely topological consequences of Caratheodory's Theory of prime ends, from "Selected Studies", (Th M Rassias, G M Rassias, editors), North-Holland (1982) 225-255

[23] S Matsumoto, Some remarks on foliated $S^{1}$ bundles, Invent. Math. 90 (1987) 343-358

[24] J Milnor, Introduction to Algebraic K theory, Annals of Mathematics Studies 72, Princeton University Press (1971)

[25] J Milnor, On the existence of a connection with curvature zero, Comm. Helv. Math. 32 (1958) 215-223 
[26] J Montesinos, Classical Tessellations and Three-Manifolds, Universitext, Springer-Verlag, Berlin (1985)

[27] R L Moore, Foundations of point set theory, American Mathematical Society Colloquium Publications, Vol. XIII Amer. Math. Soc., Providence, RI (1962)

[28] R L Moore, J Kline, On the most general plane closed point set through which it is possible to pass a simple continuous arc, Ann. Math. 20 (1919) 218-223

[29] R Mura, A Rhemtulla, Orderable groups, Lecture Notes in Pure and Applied Mathematics 27, Marcel Dekker, Inc., New York-Basel (1977)

[30] Ch Pommerenke, Boundary behaviour of conformal maps, Die Grundlehren der Mathematischen Wissenschaften 299, Springer-Verlag, Berlin (1992)

[31] R Roberts, M Stein, Exceptional Seifert group actions on $\mathbb{R}$, J. Knot Theory Ramifications 8 (1999) 241-247

[32] H Short, B Wiest, Orderings of mapping class groups after Thurston, Enseign. Math. (2) 46 (2000) 279-312

[33] E Slaminka, A Brouwer translation theorem for free homeomorphisms, Trans. Amer. Math. Soc. 306 (1988) 277-291

[34] S Smale, Diffeomorphisms of the 2-sphere, Proc. Amer. Math. Soc. 10 (1959) 621-626

[35] S Smale, The classification of immersions of spheres in Euclidean spaces, Ann. of Math. (2) 69 (1959) 327-344

[36] W Thurston, Foliations and groups of diffeomorphisms, Bull. Amer. Math. Soc. 80 (1974) 304-307

[37] W Thurston, A generalization of the Reeb stability theorem, Topology 13 (1974) 347-352

[38] W Thurston, 3-manifolds, foliations and circles II, preprint

[39] T Tsuboi, On the foliated products of class $C^{1}$, Ann. Math. 130 (1989) 227-271

[40] T Tsuboi, Homology of diffeomorphism groups, and foliated structures, Translated in Sugaku Expositions 3 (1990) 145-181

[41] H Whitney, On regular closed curves in the plane, Compositio Math. 4 (1937) 276-284

[42] J Wood, Bundles with totally disconnected structure group, Comm. Math. Helv. 46 (1971) 257-273

Department of Mathematics, California Institute of Technology

Pasadena CA, 91125, USA

Email: dannyc@its.caltech.edu

Received: 9 September 2003 Revised: 30 July 2004 